ARTICLE

\title{
Increased pathogenicity of pneumococcal serotype 1 is driven by rapid autolysis and release of pneumolysin
}

Laura C. Jacques', Stavros Panagiotou (1) 1, Murielle Baltazar (1) 1, Madikay Senghore ${ }^{2}$, Shadia Khandaker ${ }^{1}$, Rong $\mathrm{Xu}^{1}$, Laura Bricio-Moreno ${ }^{1}$, Marie Yang ${ }^{1}$, Christopher G. Dowson (1) ${ }^{3}$, Dean B. Everett (1) ${ }^{4}$, Daniel R. Neill (1) ${ }^{1} \&$ Aras Kadioglu (i) ${ }^{1 凶}$

Streptococcus pneumoniae serotype 1 is the predominant cause of invasive pneumococcal disease in sub-Saharan Africa, but the mechanism behind its increased invasiveness is not well understood. Here, we use mouse models of lung infection to identify virulence factors associated with severe bacteraemic pneumonia during serotype-1 (ST217) infection. We use BALB/c mice, which are highly resistant to pneumococcal pneumonia when infected with other serotypes. However, we observe $100 \%$ mortality and high levels of bacteraemia within 24 hours when BALB/c mice are intranasally infected with ST217. Serotype 1 produces large quantities of pneumolysin, which is rapidly released due to high levels of bacterial autolysis. This leads to substantial levels of cellular cytotoxicity and breakdown of tight junctions between cells, allowing a route for rapid bacterial dissemination from the respiratory tract into the blood. Thus, our results offer an explanation for the increased invasiveness of serotype 1.

\footnotetext{
${ }^{1}$ Department of Clinical Infection, Microbiology and Immunology, Institute of Infection and Global Health, University of Liverpool, Liverpool, UK. ${ }^{2}$ Medical Research Council Unit, The Gambia, Bakau, Gambia. ${ }^{3}$ School of Life Sciences, University of Warwick, Warwick, UK. ${ }^{4}$ Malawi-Liverpool-Wellcome Trust Clinical Research Programme, University of Malawi, College of Medicine, Blantyre, Malawi. ${ }^{凶}$ email: a.kadioglu@liverpool.ac.uk
} 
S treptococcus pneumoniae (the pneumococcus) is a major human pathogen responsible for a range of severe diseases including pneumonia, meningitis and bacteraemia ${ }^{1}$. The pneumococcus accounts for significant morbidity and mortality globally, particularly in high risk groups such as the elderly, infants and the immunocompromised ${ }^{2}$.

Serotype 1 has unusual clinical, epidemiological, genetic and microbiological characteristics, making it the most prevalent of the highly invasive pneumococcal serotypes in sub-Saharan Africa $^{3-6}$. Disease rates in the young and in otherwise healthy adults are high, whilst carriage rates are low, even when symptomatic disease is prevalent in the population ${ }^{3,7}$. Epidemiological studies have found that serotype 1 causes a large proportion of the pneumococcal disease seen in HIV-uninfected children, suggesting that serotype 1 is highly virulent and more invasive than other serotypes, enabling it to cause disease in otherwise healthy individuals ${ }^{7-10}$. Despite temporal reductions in disease incidence after the introduction of the Pneumococcal Conjugate Vaccine (PCV13) in 2011, serotype 1 remains the most common serotype causing invasive pneumococcal disease (IPD) in Southern and sub-Saharan Africa ${ }^{11}$.

The pneumococcal toxin pneumolysin is expressed by virtually all clinical isolates and has been described as a key virulence factor contributing to high morbidity and mortality rates in invasive disease $\mathrm{e}^{12-16}$. Pneumolysin is a member of the cholesterol dependent cytolysin (CDC) family of membrane-binding toxins ${ }^{17}$. It lyses cells with cholesterol in their membranes, activates host complement and induces pro-inflammatory reactions in immune cells ${ }^{18,19}$. For example, experiments in human lung tissue demonstrate that detection of pneumolysin by NLRP3 inflammasomes mediates production of inflammatory cytokines such as IL- $1 \beta$ and IL- $8^{20}$. At lytic concentrations, pneumolysin causes widespread cellular and tissue damage, allowing for increased bacterial replication and tissue invasion ${ }^{14}$. As pneumolysin is a cytosolic toxin, release is mediated by an autolysindependant process ${ }^{21}$. This process is characterised by cell wall degradation by a peptidoglycan hydrolase (autolysin), the most common of which is known as LytA ${ }^{22}$. As pneumolysin lacks an $\mathrm{N}$ terminal secretion signal sequence, lysis of the bacteria by autolysin is essential for toxin release ${ }^{21,23,24}$. Genetically designed pneumolysin-deficient (PLY-) and autolysin (LytA ${ }^{-}$) deficient mutant pneumococcal strains have been shown to be attenuated in virulence after intranasal administration into mice ${ }^{12,25-28}$. Hence, pneumolysin and autolysin work hand in hand as key pneumococcal virulence factors.

The presence of cytotoxic pneumolysin in the lung during the initial phase of pneumonia contributes to the development of bacteraemia, facilitating penetration of bacteria from the alveoli into the interstitium of the lung, and dissemination of pneumococci into the bloodstream, during experimental models of pneumonia 29,30 .

The importance of pneumolysin in pneumococcal carriage and invasive disease has been well characterised in serotype 2 (D39) infection but little is known about the combinations of key virulence factors that contribute to serotype 1 disease pathogenesis. Post vaccine studies are ongoing to monitor the effect of PCV13 introduction on serotype 1 distribution, however, an indirect cohort analysis from a surveillance programme failed to demonstrate significant protection against serotype 1 IPD by $\mathrm{PCV} 3^{31}$. In addition, phylogenetic analysis of invasive serotype 1 pneumococcal isolates in South Africa show increases in genetic diversity and an increase of lineages associated with antimicrobial non-susceptibility post-PCV13 11 . Hence, an understanding of the contribution of key virulence factors to pneumococcal pathogenesis could aid both future vaccine design and potential therapeutics for serotype 1 IPD.
To identify factors that make key contributions to serotype 1 disease pathogenesis, we used murine models of experimental pneumococcal pneumonia and nasopharyngeal carriage. Experiments were performed with the pneumococcal pneumoniaresistant $\mathrm{BALB} / \mathrm{c}$ strain of mice ${ }^{32-34}$, and disease severity of infection with African serotype 1 (ST217 and ST3081) was compared to serotypes 2 (D39), 5, 6B and 7F infection. African serotype 1 clonal complex ST217 was used, as this has been the dominant clone amongst serotype 1 isolates in Africa since $1989^{6,11}$

Here, we demonstrate that in a normally resistant BALB/c pneumonia model, African serotype 1 induced $100 \%$ mortality, whilst mice challenged with the other serotypes all survived. Serotype 1 virulence was driven by rapid bacterial autolysis, which leads to the release of large quantities of pneumolysin, enabling rapid bacterial dissemination into the bloodstream.

\section{Results}

Serotype 1 pneumococci display high tissue invasiveness. $\mathrm{BALB} / \mathrm{c}$ mice intranasally infected with $10^{6}$ colony forming units (CFU) of serotype 1 (ST217) displayed $100 \%$ mortality within $48 \mathrm{~h}$ of infection, compared with $0 \%$ mortality in mice infected with the same concentration of serotype 2 (D39) (Fig. 1a).

In addition, as early as $6 \mathrm{~h}$ post-infection, $60 \%$ of serotype 1 infected mice had significant bacteremia (mean CFU: $5 \times 10^{4} \mathrm{cfu}$ per $\mathrm{ml}$ ), increasing to $100 \%$ of mice by $12 \mathrm{~h}$ post infection until time of death (mean CFU: $3 \times 10^{6} \mathrm{cfu}$ per ml). No bacteria were detectable in the blood of serotype 2 (D39)-infected mice at any time point tested (Fig. 1b). Despite these significant differences in bacteremia, no significant differences in lung bacterial loads were observed between serotype 2 and serotype 1 -infected mice over the first $12 \mathrm{~h}$ post infection (Fig. 1c) and so we hypothesised that the level of bacterial load in lungs is not directly responsible for bacterial dissemination into the blood stream in this murine model of pneumonia.

Lung tissue from naïve and S. pneumoniae-infected mice was used to examine damage, via histopathology, during pneumococcal pneumonia (Fig. 2). Substantially greater levels of immune cell infiltration, inflammation and pathology were observed in ST217-infected lungs as compared to D39 at equivalent timepoints. By $18 \mathrm{~h}$ post-infection, lungs infected with ST217 exhibited hypertrophy of bronchiole walls, heavy cellular infiltration around bronchioles and evidence of oedema. By $24 \mathrm{~h}$ post-infection, extension of inflammatory cell infiltration from bronchioles and perivascular areas into the surrounding lung parenchyma was apparent with several focal areas of heavy consolidation becoming larger and more diffuse. Although a gradual increase in cellular infiltration and inflammation was also observed in D39-infected lungs, this was substantially less prominent than that seen in ST217-infected lungs.

Immune cell analysis of ST217 and D39 infected lungs was performed at 6, 12 and 24 hours post infection (Supplementary Figs. 1A-C, 9 and 10). Mice infected with ST217 had significantly higher numbers of $\mathrm{Gr}-1^{+}$neutrophils in the lungs $6 \mathrm{~h}$ post infection compared to D39-infected mice $(P<0.01)$ and this trend continued at 12 and $24 \mathrm{~h}$ post-infection, despite equivalent CFU levels across these timepoints in D39 and ST217-infected mice, again suggesting that the drivers of inflammation are not bacterial numbers alone. Interestingly, despite early increases in Foxp $3^{+} \mathrm{T}$ regulatory cell numbers in ST217-infected lungs, by $24 \mathrm{~h}$ post-infection D39-infected mice had significantly higher numbers of Foxp3 ${ }^{+}$cells.

Dissemination of serotype 1 (ST217) in a non-lethal murine nasopharyngeal carriage model was also investigated (Fig. 3). During a 21-day carriage experiment in $\mathrm{BALB} / \mathrm{c}$ mice, comparing 

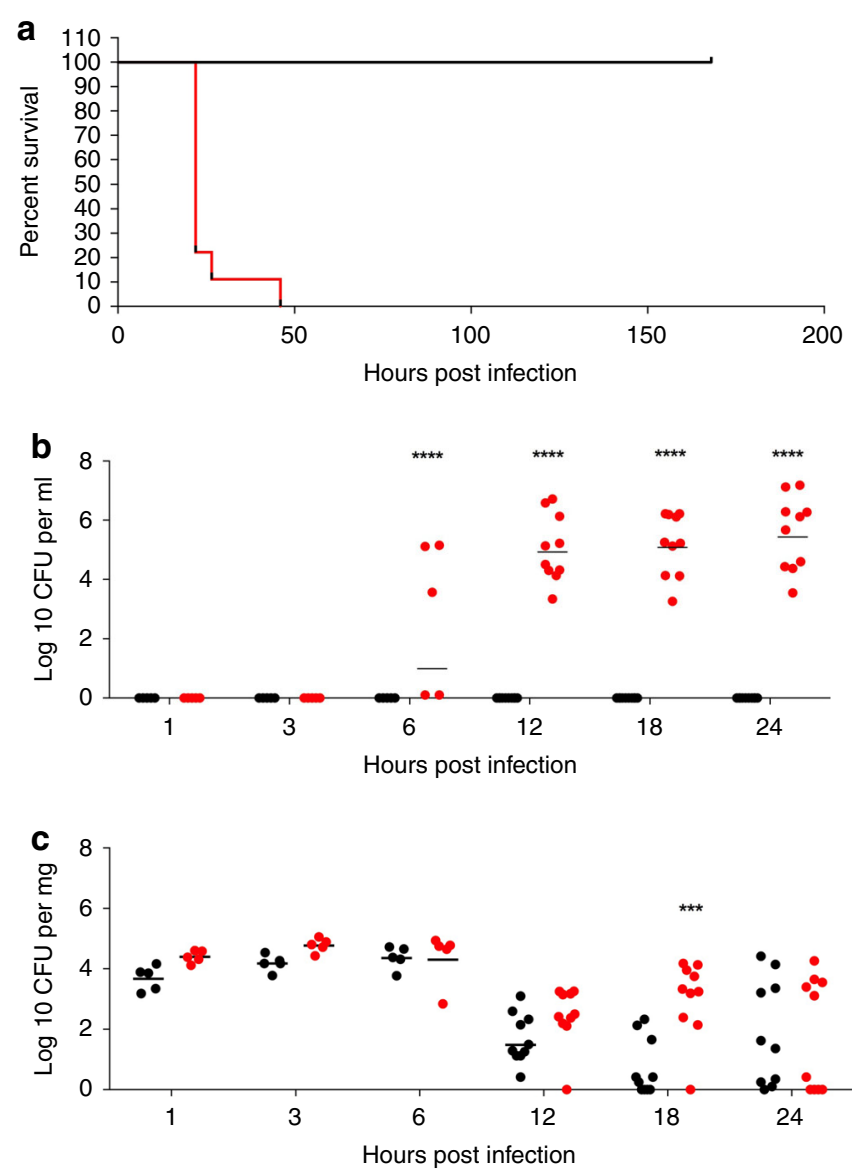

Fig. 1 Comparison of Serotype 1 (ST217S) and Serotype 2 (D39) in

BALB/c mouse pneumonia model. a Kaplan-Meier survival curve showing survival times of mice infected intranasally (IN) with $1 \times 10^{6}$ colony forming units (CFU) of serotype 1 (ST217S) (red line) or serotype 2 (D39) (black line). 1 (ST217S) infected mice $n=9$ and 2 (D39) infected mice $n=10$. b Blood bacterial load in 1 (ST217S) infected mice (red dots) and 2 (D39) infected mice (black dots) at 1,3, 6, 12, 18 and $24 \mathrm{~h}$ post intranasal infection (CFU per ml). c Lung bacterial load in 1 (ST217S) infected mice (red dots) and 2 (D39) infected mice (black dots) at 1, 3, 612,18 and $24 \mathrm{~h}$ post intranasal infection CFU per $\mathrm{mg}$ ) (each dot represents one mouse). Data are presented as follows; each symbol represents an individual mouse and geometric mean is shown by horizontal bars. Statistical analysis was performed using Two-way ANOVA and Sidaks's post-test. ${ }^{\star \star \star} P$-value $=$ $0.0001,{ }^{\star \star \star \star} P$-value $<0.0001$. Source data are provided as a Source Data file.

D39 and ST217 carriage patterns, nasopharyngeal bacterial burdens were comparable on all days except day 7 post infection when a significantly higher density of serotype 1 colonisation was detected (Fig. 3a). This higher density of serotype 1 colonisation prevailed over the next 14 days. Flow cytometry was performed to analyse the fluctuation of immune cell numbers in the nasopharynx over 21 days of colonisation in ST217-infected mice compared to D39-infected (Supplementary Figs. 1d-g, 10 and 11). Both groups of infected mice showed a similar trend of neutrophil $\left(\mathrm{CD}_{4} 5^{+} \mathrm{Gr}-1^{+}\right)$and macrophage $\left(\mathrm{CD} 45^{+} \mathrm{CD} 11 \mathrm{~b}^{+}\right.$ $\mathrm{F} 4 / 80^{+}$) numbers over time. A significant difference in both neutrophil and macrophage numbers was observed at day 14 post infection with D39-infected mice showing higher numbers of macrophages and neutrophils compared to ST217-infected mice. In addition, cells associated with the adaptive immune response were also monitored over 21 days in the nasopharynx. No significant differences in the numbers of Foxp $3^{+} \mathrm{T}$ regulatory cells or ROR $\gamma \mathrm{T}^{+}$Th17 cells were observed between ST217 and D39 colonisation. In addition, no significant differences were observed in the number of Foxp $3^{+}$cells expressing TGF $\beta$ or Th17 cells expressing IL-17A between infection with the different serotypes.

Of note, serotype 1 was able to invade and colonise the olfactory tissues at a substantially higher density over 14 days than D39, with significantly higher ST217 CFU at days 5 and 7 post infection in olfactory epithelia and bulb respectively (Fig. 3b, c). Importantly, while serotype 1 colonies were detected in the brain during the first 7 days of nasopharyngeal carriage, no D39 colonies were detected throughout this experiment (Fig. 3d), demonstrating the capacity of serotype 1 to translocate across nasopharyngeal to olfactory and ultimately brain tissue.

Neither ST217, nor D39, colonies were detected in the blood at any timepoint (data not shown), suggesting that serotype 1 may utilise host invasion of olfactory tissues to enter the brain, bypassing the blood-brain barrier. Indeed, direct invasion from the nasopharynx to the brain via the olfactory tissues has been previously reported for serotype 19F, in immunocompromised XID mice that are highly susceptible to pneumococcal infection ${ }^{35}$. To the best of our knowledge, this is the first reported description of this pathway of translocation from nasopharyngeal tissues to meninges for Streptococcus pneumoniae in immunocompetent mice.

Comparison of invasive clinical isolates in a pneumonia model. To assess whether high mortality rates in resistant $\mathrm{BALB} / \mathrm{c}$ mice were unique to serotype 1 (ST217) infection, the pneumonia survival model was repeated with different clinical isolates of $S$. pneumoniae (Fig. 4a). The isolates tested included the invasive clinical serotypes $1,5,7 \mathrm{~F}$ and $6 \mathrm{~B}$, all of which are included in the current PCV13 vaccine ${ }^{36}$. Additional serotype 1 isolates were also included in these experiments, including another ST217 isolate from Karonga, Malawi (ST217C), an ST3081 from the Gambia and a European serotype 1 isolate (ST306) expressing a nonhaemolytic pneumolysin. Strikingly, while $100 \%$ mortality was observed in $\mathrm{BALB} / \mathrm{c}$ mice infected with all African serotype 1 isolates (ST217S, ST217C and ST3081), 100\% survival was seen with all other serotypes tested, including the non-haemolytic serotype 1 strain ST306 (Fig. 4a).

Comparison of pneumolysin production in clinical isolates. ST306 expresses a non-haemolytic pneumolysin and was the only serotype 1 sequence type not to cause any mortality in vivo, further suggesting that pneumolysin is a key determinant of serotype 1 virulence in vivo. The amount of pneumolysin released upon total lysis of $10^{7} \mathrm{CFU}$ of different pneumococcal serotypes was determined by ELISA (Fig. 4b). Total pneumolysin content of lysed CFU varied considerably between isolates, from $20 \mathrm{ng}$ per $\mathrm{ml}$ for serotype 19F to over $400 \mathrm{ng}$ per ml for serotype 1 (ST306) (Fig. 4b). Serotype 1 isolates produced between 200 and $420 \mathrm{ng}$ of pneumolysin upon lysis, a range of production at the upper end of the strains tested, and significantly more than that produced by D39, but comparable with that of the non-lethal serotype 5 and 7F strains.

In order to determine whether pneumolysin was one of the factors responsible for ST217 virulence, we replaced the ply gene of D39 with that of ST217. Mice infected with D39:ST217ply showed a phenotype intermediate between those infected with D39 and those infected with ST217. We observed 50\% mortality in D39:ST217ply infected mice (Fig. 4a, Supplementary Fig. 2a) and bacteraemia was observed as early as $3 \mathrm{~h}$ post-infection (Supplementary Fig. 2b). However, by $24 \mathrm{~h}$ post-infection, only $50 \%$ of mice remained bacteraemic (Supplementary Fig. 2b), compared with $100 \%$ of ST217-infected animals (Fig. 1b). D39: 


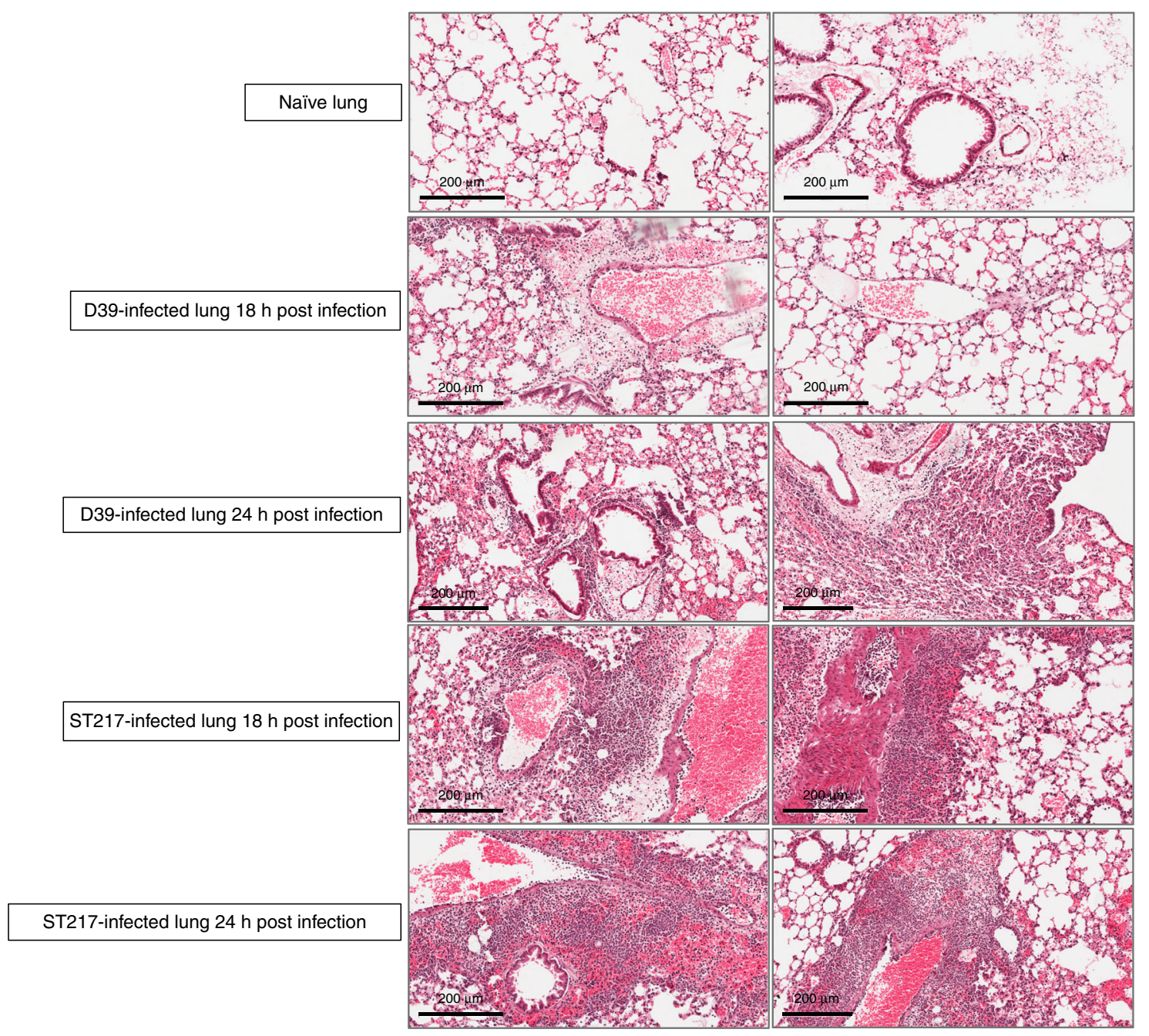

Fig. 2 Lung histopathology during murine pneumonia infection with Serotype 1 (ST217) and Serotype 2 (D39) S. pneumoniae. Mice were infected as described in Fig. 1. Lung sections from infected mice were stained with haematoxylin and eosin to show changes in lung pathology during pneumococcal infection with either 1 (ST217S) or 2(D39) at 18 and $24 \mathrm{~h}$ post infection ( 5 mice/group/timepoint). Lung sections from naïve mice ( $n=5$ ) are also shown. Scale bars $=200 \mu \mathrm{m}$.

ST217ply expressed levels of pneumolysin that were significantly higher than D39 and comparable to those of ST217 (Fig. 4b), suggesting that virulence of ST217 is at least partially due to high levels of pneumolysin production.

Significantly higher autolysis rates found in Serotype 1 isolates. The release of pneumolysin depends upon autolysin-dependent lysis of pneumococci ${ }^{37}$. As autolysis is a key determinant of toxin release, measurements of bacterial autolysis were used to assess the amount of pneumolysin released from the cytoplasm of the cells.

Triton-X induced autolysis assays were performed to assess the relative rates of autolysis amongst different pneumococcal isolates with moderate to high levels of pneumolysin production (Fig. 4c and Supplementary Fig. 3). A D39 LytA knockout mutant (LytA ${ }^{-}$) was used as a control in these experiments to show that Triton-X induced autolysis was dependent on LytA activation. $\mathrm{OD}_{600}$ readings significantly decreased (shown as percentage increase in autolysis) for serotype 1; ST3081, ST217C, ST217S and D39: ST217ply strains $60 \mathrm{~min}$ after Triton $\mathrm{X}$ treatment compared to serotype 2 (D39). Interestingly, although serotype 5 produced pneumolysin levels comparable with those of serotype 1 , it caused no mortality in the BALB/c pneumonia model and rates of Triton-X induced autolysis were found to be significantly lower in serotype 5 compared to serotype 1 isolates after 60 min of Triton $\mathrm{X}$ treatment. Consistent with its intermediate virulence phenotype, the D39 strain expressing the ST217 pneumolysin (D39: ST217ply) had an autolysis rate that was significantly higher than that of the parental D39 strain but lower than that of the serotype 1 isolates. Serotype 5 and the D39:ST217ply strain had initially high autolysis rates but these plateaued between 60 and $120 \mathrm{~min}$ post Triton X treatment (Supplementary Fig. 3). These findings suggest that autolysis is significantly more rapid in serotype 1, leading to enhanced release of pneumolysin, as compared to other serotypes.

Serotype 1 supernatant aids virulence of D39 in pneumonia model. To examine whether natural rates of autolysis (not induced by Triton-X) might contribute to in vivo virulence, and to determine whether extracellular pneumolysin within the supernatant of the infection inoculum might impact on disease progression, a supernatant switch experiment was designed. Serotype 1 (ST217S) and serotype 2 (D39) inoculum doses $\left(2 \times 10^{7}\right.$ CFU each) were prepared in $1 \mathrm{ml}$ of PBS. The doses were 
a
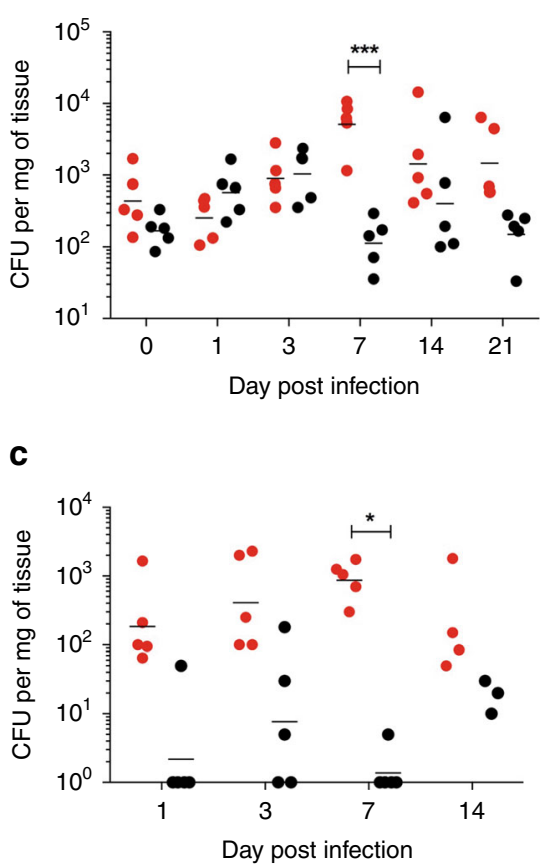

b

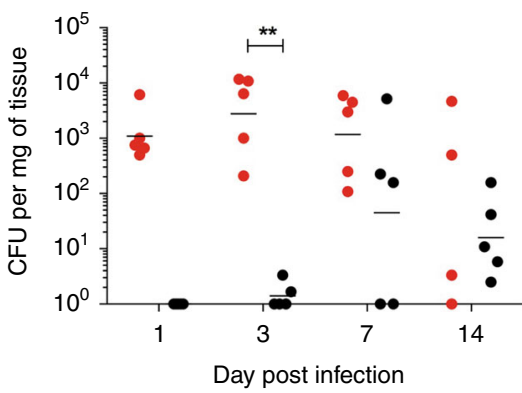

d

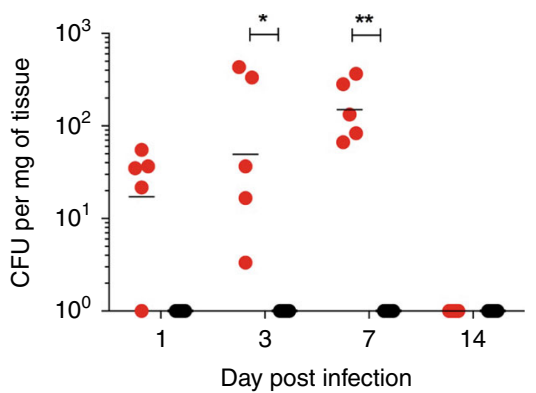

Fig. 3 Comparison of Serotype 1 (ST217S) and Serotype 2 (D39) in BALB/c mouse nasopharyngeal carriage model. Mice were infected by intranasal administration with either $10^{5}$ CFU of 1(ST217S) or 2(D39) and culled at 0,1, 3, 7, 14 or 21 days post infection. In all, 5-10 mice per group were culled and tissues were taken for analysis of bacterial load. Data presented as follows; each symbol represents an individual mouse and error bars show the geometric mean. Mice infected with 1 (ST217S) are shown by red dots and 2(D39) infected shown as black dots. Statistical analysis was performed using Two-way ANOVA and Sidaks comparison test. a CFU counts in the nasopharynx of infected mice. ${ }^{\star \star \star} P$ value $=0.0008$ (b) CFU counts in the Olfactory epithelium of infected mice. ${ }^{\star \star} P$ value $=0.0025$. c CFU counts in the Olfactory Bulb of infected mice. ${ }^{\star} P$ value $=0.0341$. $\mathbf{d}$ CFU counts in the brain of infected mice. ${ }^{\star} P$ value $=0.0200,{ }^{\star \star} P$ value $=0.0070$. Source data are provided as a Source Data file.

incubated for $60 \mathrm{~min}$ at room temperature. An ELISA-based detection method was used to measure the concentration of pneumolysin released into the inoculum dose during this time period (the supernatant). PLN-A (isogenic pneumolysin-negative mutant of D39) was used as a pneumolysin-negative control. Results demonstrate that the serotype 1 inoculum had significantly higher concentrations of pneumolysin in its supernatant compared to the D39 supernatant $(* * * P=0.0002$; Fig. 5a). To investigate the effect of high concentrations of supernatant pneumolysin in the serotype 1 inoculum dose on induction of disease, the supernatants of serotype 1 and D39 inoculum doses were swapped before infection of the mice. D39 and serotype 1 infection doses were prepared in $1 \mathrm{ml}$ of PBS, incubated at room temperature for $60 \mathrm{~min}$ and then immediately prior to infection, bacteria were pelleted by centrifugation and the supernatants of the two doses were swapped. Mice were infected with either D39 bacteria resuspended in supernatant from serotype 1 (ST217S) inoculum dose or serotype 1 (ST217S) bacteria resuspended in supernatant from D39 inoculum dose.

High concentrations of pneumolysin in the supernatant of inoculum doses contributed significantly to mortality in the mouse pneumonia model (Fig. 5b). When mice were infected with serotype 1 bacteria and serotype 1 supernatant, $100 \%$ mortality was observed with a mean survival time of $25 \mathrm{~h}$ compared to $100 \%$ survival in mice infected with D39 bacteria in D39 supernatant. When mice were infected with D39 bacteria with serotype 1 supernatant, survival rates decreased from $100 \%$ to $40 \%$, with a mean survival time of $62.5 \mathrm{~h}$, and when mice were infected with serotype 1 bacteria with D39 supernatant, mean survival time increased from $25 \mathrm{~h}$ to $48 \mathrm{~h}$ and $20 \%$ survival rates were observed. Finally, when serotype 1 supernatant was pretreated with cholesterol-containing liposomes (which we have previously shown to strongly bind and sequester pneumolysin) ${ }^{38}$ prior to resuspension of the D39 pellet and infection of mice, survival rates returned to $100 \%$, confirming that the serotype 1 pneumolysin in the supernatant is responsible for increased mortality in D39-infected mice.

Cytotoxic effects of serotype 1 pneumolysin on lung epithelial cells. Excess concentrations of pneumolysin in the lung have previously been shown to aid bacterial replication and increase rates of tissue invasion and dissemination into the bloodstream $^{14,30}$. Lactate dehydrogenase $(\mathrm{LDH})$ assays were used to measure cytotoxicity of A549 human lung epithelial cells upon infection with a range of clinical serotypes of $S$. pneumoniae in vitro. Infection with serotype 1 (ST217S) caused significantly more cytotoxic damage to lung epithelial cells compared to other serotypes at equivalent CFU load (Fig. 6). At $6 \mathrm{~h}$ post infection, lung epithelial cells cultured with serotype 1 (ST217S) had significantly higher levels of $\mathrm{LDH}$ compared to infection with serotype 2 (D39), 5 and 7F. LDH levels were also significantly higher at $12 \mathrm{~h}$ post infection with ST217S compared to infection with serotype $5,6 \mathrm{~B}$ and 2 (D39).

Serotype 1 pneumolysin damages epithelial tight junctions. Serotype 1 is able to disseminate from the lungs into blood as early as $6 \mathrm{~h}$ post infection in vivo (Fig. 1b), leading to systemic infection and high mortality. It also causes early and sustained $\mathrm{LDH}$ release from lung epithelial cells in vitro, suggesting that translocation from infected lungs into blood in vivo is most likely due to damage to lung epithelial cell barriers. To further investigate this, in vitro assays were performed to measure the barrier function of human primary pulmonary alveolar cells (Fig. 7) and A549 epithelial cells (Supplementary Fig. 4) during infection with 


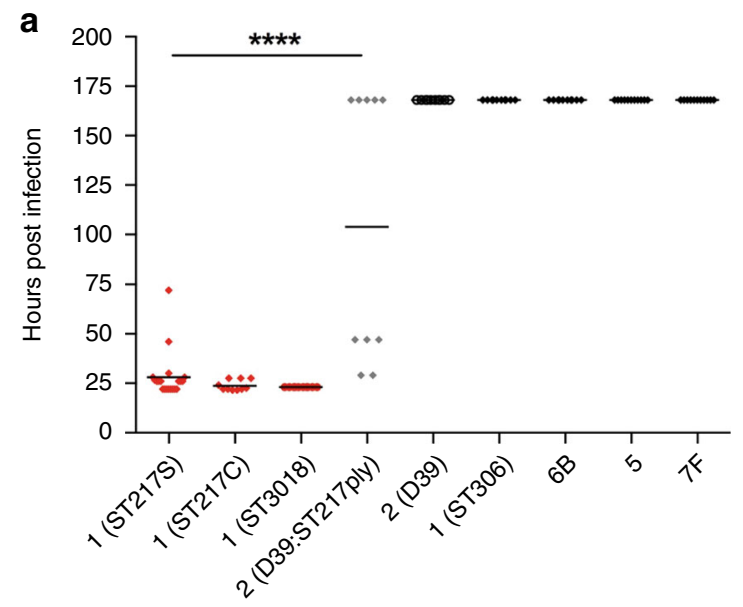

b
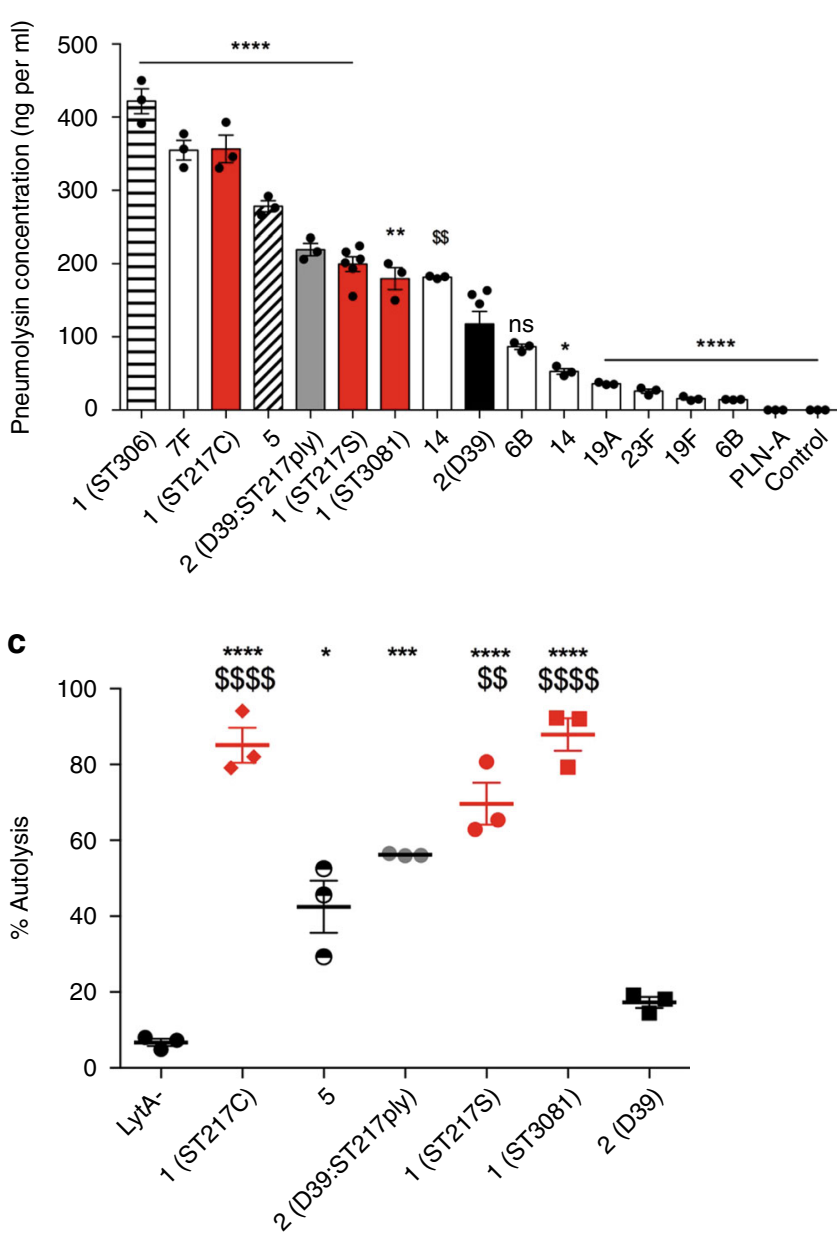

a range of clinical serotypes. A monolayer of cells was infected with S. pneumoniae, and trans-epithelial electrical resistance (TEER) measurements taken as an indicator of barrier integrity (Fig. 7). TEER readings are used as an indicator of the permeability of tight junctions between cells grown in culture ${ }^{39}$ and decrease in the TEER value is an indicator of disruption or damage of tight junctions. Infection of both primary and immortalised lung epithelial cells with serotype 1 (ST217) resulted in a significant drop in TEER compared to infection with other isolates of $S$. pneumoniae (D39, 5 and ST306; Fig. 7a-c).
Fig. 4 A combination of pneumolysin production and rates of autolysis correlate with pneumococcal virulence. a Mice were infected with $1 \times 10^{6}$ colony forming units (CFU) S. pneumoniae by intranasal administration and monitored closely for signs of ill-health. If infected mice became lethargic, they were culled, survival times noted and CFU from blood determined by Miles and Misra dilution. Survival times of mice infected with African serotype 1 strains (red) compared to other clinically relevant strains (black) and a D39 strain expressing the ST217S pneumolysin (grey) (10 mice/ group). Experiment was ended at $168 \mathrm{~h}$. Statistical analysis was performed using One-way ANOVA and Dunnett's multiple comparisons test:

${ }^{\star \star \star \star} P$-value $<0.0001$ vs D39. $\mathbf{b}$ ELISA-based quantification of the amount of pneumolysin released by different strains and serotypes ( $10^{7} \mathrm{CFU}$ ) upon lysis by penicillin/streptomycin treatment. Data represented as mean \pm SEM. For all serotypes $n=3$ biologically independent samples, whereas for 1(ST217S) and 2(D39), $n=6$ are shown (six biologically independent samples over two independent experiments). Statistical analysis was performed by one-way ANOVA with Dunnett's multiple comparison test. Asterisk represent significant differences versus serotype 2 (D39). ${ }^{\star} P<0.0132,{ }^{\star \star} P<0.01,{ }^{\star \star \star \star} P<0.0001$, ns $=$ not significant. c Bacteria $\left(\mathrm{OD}_{600} 1.0\right.$ ) were incubated at $37^{\circ} \mathrm{C}$ and $175 \mathrm{rpm}$ with $0.01 \%$ Triton X. At 60 min post treatment, $\mathrm{OD}_{600}$ was measured and converted to a percentage of the original $\mathrm{OD}_{600}$ reading. Percentage autolysis represents the percentage decrease of original $\mathrm{OD}_{600}$ reading. Statistical analysis was performed by Two-way ANOVA and Tukey's multiple comparisons test. Rates of autolysis were measured in triplicates; data are presented as mean \pm SEM. Asterisk represent comparisons of serotype 1 to 2(D39) and \$ represents comparisons of serotype 1 to serotype 5 . ( $\mathrm{ns}=$ non significant. $\left.{ }^{\star} P=0.0103, \$ \$ P=0.0056,{ }^{\star \star \star} P=0.0002,{ }^{\star \star \star *} / \$ \$ \$ P<0.0001\right)$. Source data are provided as a Source Data file.

The addition of liposomes, to bind and sequester pneumolysin released from ST217, resulted in significantly higher TEER measurements and therefore less damage to the epithelial barrier at $24 \mathrm{~h}$ post infection (Fig. $7 \mathrm{~d}$ ).

In addition to performing TEER readings on infected HPAEpiCs, the permeability of the tight junctions was assessed using FITC-Dextran (Fig. 8), as well as monitoring the expression of zonula occludens protein 1 (ZO-1) during infection (Fig. 9). Significantly higher emissions of FITC-dextran were observed on the basolateral side of HPAEpiCs infected with serotype 1 (ST217) compared to other serotypes, confirming our findings with TEER readings that suggested that high concentrations of pneumolysin cause significant damage to epithelial cell tight junctions. This effect was significantly reduced in the presence of liposomes. Cell damage by ST217 was independently confirmed using an MTT assay (Supplementary Fig. 5).

ZO-1 protein localizes at tight junction sites in epithelial and endothelial cells and fluorescent staining of this protein can be used to visualise cellular tight junctions ${ }^{40}$. Infection with D39 or ST217 induced markedly decreased abundance in total ZO-1 protein as compared to uninfected and ST306-infected cells (Fig. 9a, b). This effect was most pronounced in ST217-infected cells. There was also evidence of decreased association of ZO-1 with epithelial membranes in ST217-infected cells (Fig. 9a).

\section{Discussion}

Streptococcus pneumoniae serotype 1 is the most prevalent and invasive pneumococcal serotype in sub-Saharan Africa, although the bacterial factors driving pathogenesis are not well understood. Here, we used murine infection models and in vitro assays to identify key virulence determinants associated with increased invasiveness of serotype 1 .

Previous published work has demonstrated that $\mathrm{BALB} / \mathrm{c}$ mice are highly resistant to invasive pneumococcal pneumonia when 

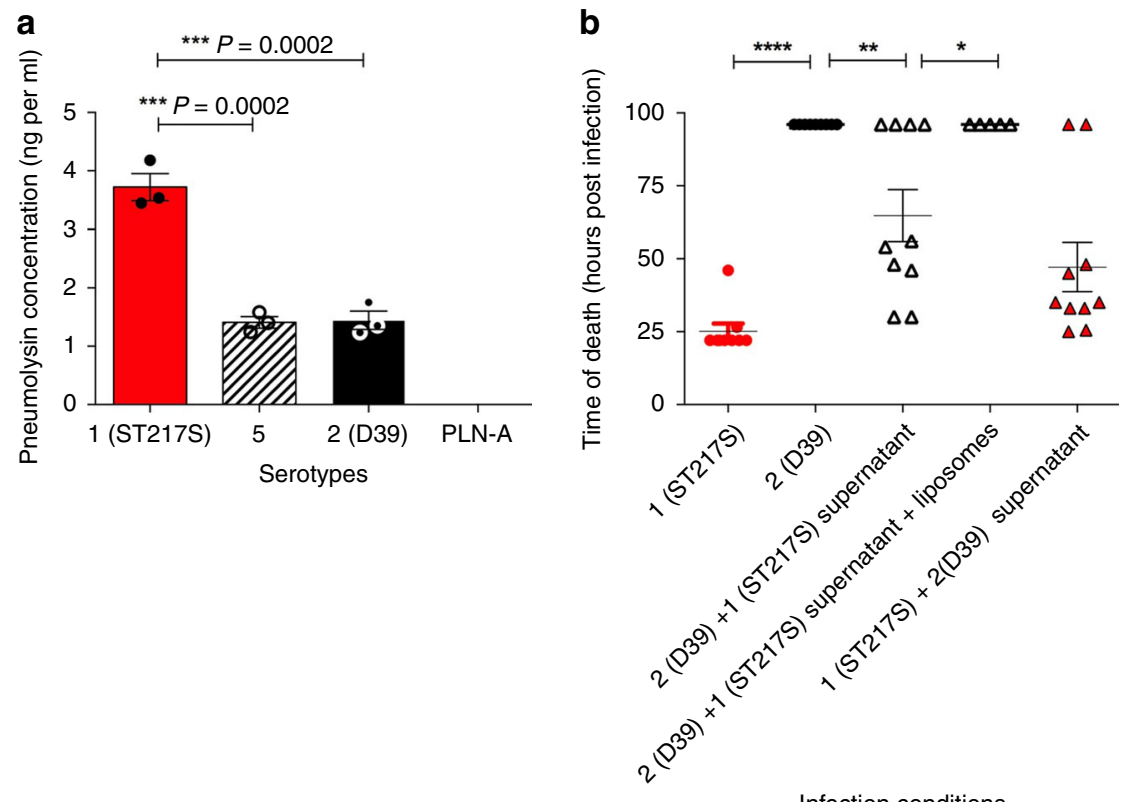

Infection conditions

Fig. 5 High concentration of pneumolysin in infection dose correlates with high mortality rates in BALB/c mice. a In separate tubes, $2 \times 10^{7} \mathrm{CFU}$ per $\mathrm{ml}$ of 1 (ST217S), 2(D39), serotype 5 and PLN-A were prepared in PBS. Dose preparations were incubated at room temperature for 60 min before a sample of supernatant was removed and pneumolysin detection ELISA used to quantify the amount of pneumolysin released into the supernatant for each strain. Experiments were performed in triplicate and error bars represent mean \pm SEM. Statistical analysis was performed using a one-way ANOVA and Tukey's multiple comparison test. b Survival times of mice infected with different combinations of $10^{6}$ CFU of either serotype 1 (ST217S) or serotype 2 (D39) S. pneumoniae in $50 \mu \mathrm{l}$ bacterial dose supernatant that had been incubated at room temperature for $60 \mathrm{~min}, 10$ mice per group. For one group of mice, the supernatant from ST217S was treated with liposomes (a combination of $2 \mu \mathrm{g} / \mathrm{ml}$ Cholesterol:sphingomyelin ( 66 mol/\%cholesterol) and sphingomyelinonly liposomes) for $30 \mathrm{~min}$. Each symbol represents one mouse. Errors bars represent mean $\pm \mathrm{SEM}$. Statistical analysis was performed using One-way ANOVA and Tukey's multiple comparison test: ${ }^{\star} P$-value $=0.0398,{ }^{\star \star} P$-value $<0.0093$ and ${ }^{\star \star \star \star} P$-value $<0.0001$. Source data are provided as a Source Data file.

intranasally infected with serotype 2 (D39). No bacterial dissemination from lungs into bloodstream is observed, and bacteria are cleared from the lungs within 3-5 days, resulting in $100 \%$ survival rates ${ }^{32,34,41}$. However, our findings show that when $\mathrm{BALB} / \mathrm{c}$ mice were intranasally infected with African serotype 1 (ST217 and ST3018) isolates, 100\% mortality was observed as a result of high levels of bacteremia occurring within $24 \mathrm{~h}$ post infection. Indeed, dissemination of serotype 1 from lungs into bloodstream occurred as early as 6 hours post infection with ST217S, demonstrating the highly invasive capacity of serotype 1, even in normally resistant hosts. This invasive phenotype was unique to serotype 1 , as infection with other clinical serotypes did not lead to host death. The effect was confirmed to be dependent upon pneumolysin, as a D39 mutant in which the serotype 2 ply was replaced with that of serotype 1 ST217 was able to translocate from lungs to blood (just as ST217 did) and cause 50\% mortality in $B A L B / c$, as well as expressing levels of pneumolysin that were significantly higher than D39 but comparable to those of ST217.

Sequence comparisons of D39 and ST217 ply showed 6 synonymous single-nucleotide polymorphisms (SNPs) in the ST217 pneumolysin gene compared to D39 (Supplementary Fig. 6). Despite these SNPs not leading to amino acid substitutions, mRNA prediction software (RNAfold Web) suggested that the SNPs observed could alter the secondary structure of ST217 ply compared to D39 ply (Supplementary Fig. 7). These structural differences in ST217 ply mRNA may account for the increased levels of pneumolysin produced by ST217 and by the D39 mutant expressing ST217 ply.

The initial phase of pneumonia is marked by flooding of the alveoli with bacteria, but very few inflammatory cells ${ }^{42}$. Subsequent breaching of endothelial barriers is thought to facilitate pneumococcal bacteremia and is commonly associated with high mortality rates ${ }^{43}$. This often occurs in the absence of a significant inflammatory response, suggesting that this rapid dissemination is likely to be driven by bacterial toxin-induced damage of the lung epithelial barriers. We demonstrate here that different pneumococcal serotypes produce varying concentrations of pneumolysin at equivalent CFU. However, the amount of pneumolysin produced by serotype 1 was not always significantly greater than that produced by other serotypes, suggesting that it is not simply the amount of pneumolysin produced that determines virulence, but also the rate at which this pneumolysin is released.

Release of pneumolysin is dependent on the activation of autolysin (LytA) which causes rapid cell wall breakdown and cell lysis ${ }^{22,27,28}$. Hence, autolysis is key to enabling release of haemolytic pneumolysin to quickly compromise host defences before a robust immune response can be generated. Indeed, autolysin (LytA-) deficient pneumococcal strains have been shown to be less virulent after intranasal administration into mice $12,27,28$. However, an often overlooked factor is the role played by the rate of autolysis in determining disease progression. A case in point is serotype 5 , which produces a high quantity of toxin, but which does not cause mortality or disseminate into the bloodstream during pneumonia in BALB/c mice. Notably, autolysis is significantly slower in serotype 5 than in African serotype 1 isolates, suggesting that pneumolysin release by serotype 5 may not be rapid enough to avoid detection or overcome host defenses in vivo. Although the D39 strain expressing ST217 pneumolysin (D39:ST217ply) showed initially high rates of autolysis compared to the D39 parental strain, this effect did not last beyond 120 minutes post Triton-X treatment. Hence, despite D39:ST217ply exhibiting elevated autolysis rates relative to D39 (albeit 
transient), overall, it had reduced autolysis rates relative to other serotype 1 isolates, which may explain why the D39: ST217ply strain showed an intermediate virulence phenotype in vivo.

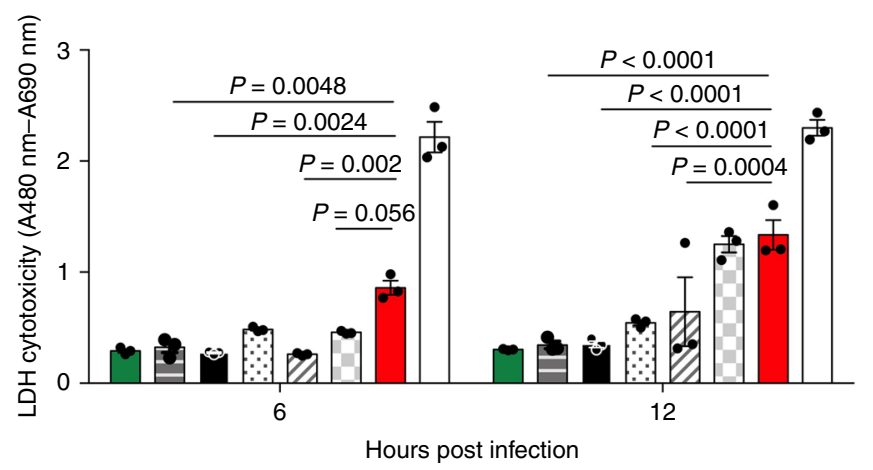

Fig. 6 Level of lung epithelial cell cytotoxicity during infection varies with serotype. A549 lung epithelial cells were infected with $6 \times 10^{5}$ CFU S. pneumoniae and, at 6 and $12 \mathrm{~h}$ post infection, samples of cell culture supernatant were removed and amount of LDH (an indicator of cytotoxic damage) measured. Infections were performed in triplicate wells and error bars represent the mean \pm SEM from three independent experiments. Bars, from left to right, are non-infected control cells (green), serotype 1 (ST306) infected (horizontal bars), 2(D39) infected (black), 6B infected (dotted bars), 5 infected (diagonal bars), 7F infected (grey squared bars) and 1 (ST217S) infected (red bars). White bars show the LDH positive control. Statistical analysis was performed using Two-way ANOVA and Dunnett's multiple comparison test. Source data are provided as a Source Data file.
This is supported by observations made in vitro, when different bacterial cultures were incubated with both A549 lung epithelial cells and primary human pulmonary alveolar epithelial cells. When comparing A549 cell infection with serotype 1 (ST217) to other clinical serotypes, significantly higher levels of epithelial cell cytotoxicity (quantified by supernatant LDH levels) was observed as early as $6 \mathrm{~h}$ post infection, suggesting that rapid release of pneumolysin by African serotype 1 causes high cellular cytotoxicity to lung epithelial cells in the context of pneumonia infection. In addition, compared to other serotypes tested, ST217 caused significant damage to human primary lung alveolar epithelial cell barriers, as measured using transepithelial electrical resistance readings (TEER) and FITC-Dextran permeability assays. This damage was significantly reduced by $24 \mathrm{~h}$ post infection with the addition of liposomes, which bind to and sequester pneumolysin. Significantly greater damage to epithelial cell barriers and increases in cellular cytotoxicity by pneumolysin released by serotype 1 isolates are likely to be the main causes of bacterial dissemination observed from lungs into blood and from nasopharynx to brain, in murine models of infection.

Additionally, ZO-1 protein was significantly reduced when lung epithelial cells were infected with ST217 or D39, but not ST306, confirming the impact of haemolytic-pneumolysin expressing serotypes on epithelial tight junctions. ZO-1 is part of a zonula occludens complex (ZO-1, ZO-2, ZO-3), and it is the first to be expressed during the formation of intercellular tight junctions ${ }^{44}$. The loss of ZO-1 was most pronounced in ST217infected cells, in line with our previous findings that this sequence type rapidly releases pneumolysin. The breakdown of this anchor protein leads to the dissociation of the cell actin cytoskeleton,

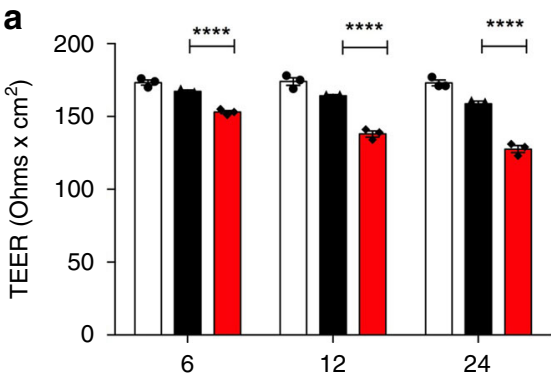

Hours post infection

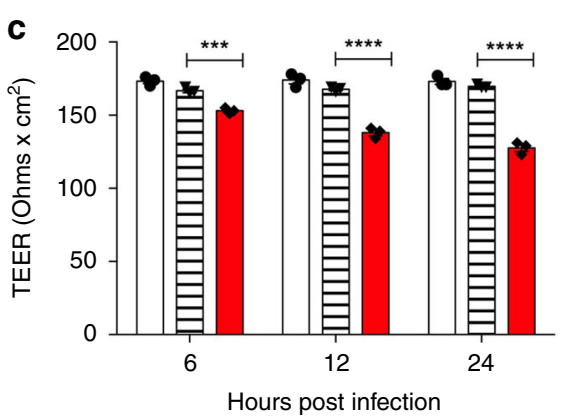

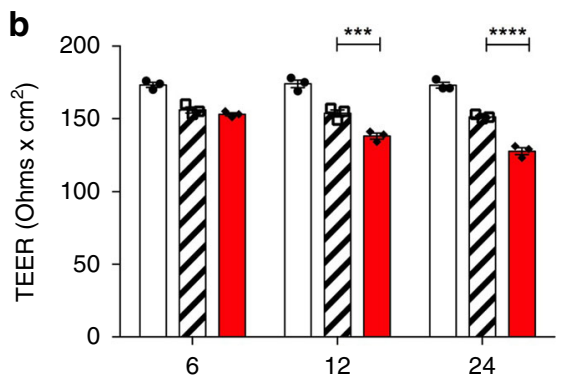

Hours post infection

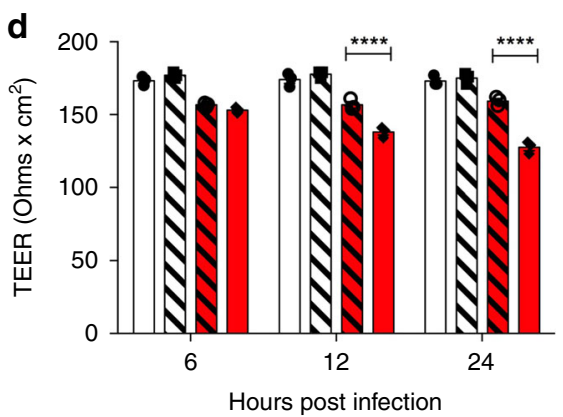

Fig. 7 African Serotype 1 causes significant damage to epithelial cell barriers. Human primary alveolar epithelial cells (HPAEpiCs) were cultured on trans-well inserts for 3 days to establish a monolayer. In all, $10^{5} \mathrm{cfu}$ of $\mathrm{S}$. pneumoniae were added and trans-epithelial electrical resistance readings (TEER) were taken at 6, 12 and $24 \mathrm{~h}$ post infection to assess the damage to tight junctions between epithelial cells. White bars represent control uninfected wells. a Comparison between serotype 1 (ST217S) (red bars) and serotype 2 (D39) (black bars) infection. ${ }^{\star \star \star \star} P$-value $<0.0001$. b Comparison between 5 (diagonal hashed bars) and serotype 1 (ST217S). ${ }^{\star \star \star} P$-value $=0.0003,{ }^{\star \star \star \star} P$-value $<0.0001$. c Comparison between serotype 1 (ST306) (horizontal striped bars) and serotype 1 (ST217S). ${ }^{\star \star \star} P$-value $=0.0001,{ }^{\star \star \star \star} P$-value $<0.0001$. d Comparison between serotype 1 (ST217S) (red bars) and serotype 1 (ST217S) with the addition of liposomes (red diagonally hashed bars). Clear, diagonally hashed bars represent cells treated with liposomes only. ${ }^{\star \star \star \star} P$-value $<$ 0.0001 . TEER readings were performed in triplicate from duplicate wells from three independent experiments. All statistical analysis was performed using Two-way ANOVA and Tukey's multiple comparison test. Error bars represents the SEM. Source data are provided as a Source Data file. 

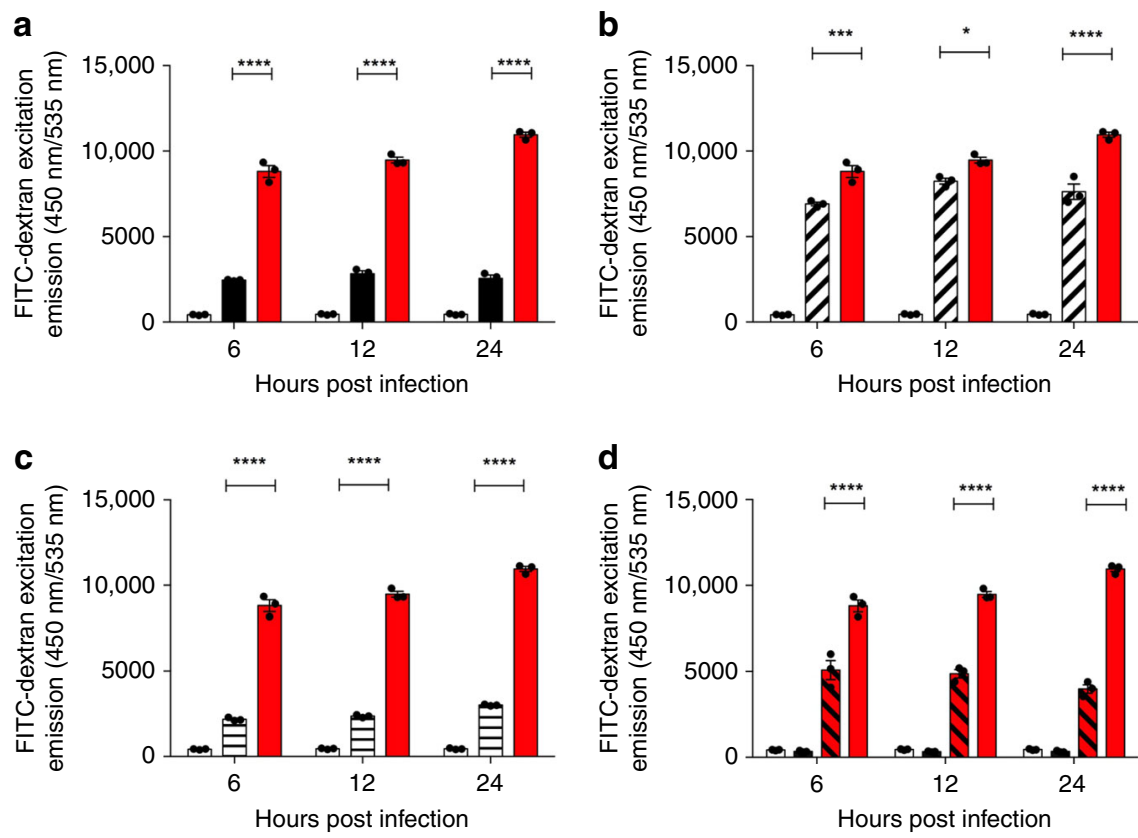

Fig. 8 African Serotype 1 causes significant increases to human primary alveolar epithelial cell barrier permeability. Assessment of epithelial cell barrier permeability changes during infection with different S. pneumoniae serotypes was performed using fluorescein isothiocyanate (FITC)-dextran (70 kDa, 1 mg per $\mathrm{ml}$ ), see methods for full details. White bars represent FITC-Dextran emissions from untreated cells. Error bars represent mean \pm SEM. Statistical analysis was performed using Two-way ANOVA and Tukey's multiple comparison test. a Comparison of FITC-Dextran excitation emission between serotype 2(D39) (black bars) and 1 (ST217S) (red bars). ${ }^{\star \star \star \star} P$-value $<0.0001$. b Comparison of FITC-Dextran excitation emission between serotype 5 (diagonally hashed bars) and 1 (ST217S) (red bars). ${ }^{\star} P$-value $=0.0178,{ }^{* \star *} P$-value $=0.0007,{ }^{\star \star *} * P$-value $<0.0001$. c Comparison of FITC-Dextran excitation emission between serotype 1 (ST306) (horizontal bars) and 1 (ST217S) (red bars). ${ }^{\star \star \star \star} P$-value $<0.0001$. d Comparison of FITC-Dextran excitation emission between serotype 1 (ST217S) infection with (red, diagonally hashed bars) and without (red bars) the addition of liposomes. ${ }^{\star \star \star \star} P$-value $<0.0001$. As a control, cells were also treated with liposomes in the absence of bacteria (black bars, second column). Source data are provided as a Source Data file.

which may provide a route for paracellular invasion of epithelial cells by ST217, hence aiding its invasiveness.

Based on our findings, we propose that the relationship between the amount of toxin produced and its rate of autolysisdependent release is a key determinant of invasiveness. The combination of these factors drive and control serotype 1 virulence. The proposed mechanism of virulence is explained in summary Fig. 10. Upon entry into the lungs, serotype 1 is likely to undergo rapid autolysis. Increased rates of autolysis will result in rapid release of high concentrations of pneumolysin, which are toxic to lung epithelial cells and cause breakdowns in tight junctions between cells. Increased cellular cytotoxicity and cell permeability allows for bacterial dissemination from lungs into the blood stream where disease progresses to bacteraemia and results in high mortality levels.

The factors governing in vivo autolysis rates largely remain elusive, but may include contributions from the extra-host environment. Indeed, the contribution of environmental factors to African serotype 1 autolysis was highlighted in a recent study, which found that increases in temperature dramatically increased rates of pneumococcal autolysis, leading to increased release of pneumolysin and higher attack rates (invasiveness) of pneumococci colonising the nasopharyn $x^{45}$. Given the inherently high rates of autolysis described here for African serotype 1, its sensitivity to higher temperatures may make a significant contribution to invasive serotype 1 disease in sub-Saharan Africa. Epidemiological studies of countries in the African meningitis belt have shown that $S$. pneumoniae serotype 1 causes up to $79 \%$ of meningitis cases among older children and working age adults $^{46}$. Data from our murine nasopharyngeal carriage studies demonstrate that serotype 1 disseminates into olfactory tissue and subsequently brain tissue during asymptomatic carriage; an event not observed during serotype 2 colonisation. During infection, rapid autolysis and release of pneumolysin by serotype 1 may enable bacterial dissemination from the nasopharyngeal tissues to the brain via the olfactory bulb. This phenomenon may contribute to the high burden of serotype 1 pneumococcal meningitis in the African meningitis belt.

The potency of free/released pneumolysin in supernatants taken from serotype 1 ST217 inoculum doses was clearly demonstrated when these supernatants were used to drive increased invasiveness of serotype 2 (D39) in a mouse pneumonia model. Addition of ST217 supernatant was sufficient to increase mortality in D39 infection from 0 to $60 \%$. These results demonstrate that serotype 1 pneumolysin is key in driving bacterial dissemination from the lungs into blood, resulting in high mortality rates. Furthermore, D39, which produces lower quantities of pneumolysin, is capable of significantly increased virulence and proliferation in blood if aided across the lung epithelial barrier by serotype 1 pneumolysin, suggesting that penetration of the lung epithelial barrier is the key step in the progression to bacteremia and lethality. Enhanced virulence of D39 when combined with ST217 supernatant was completely reversible with the addition of liposomes that bound and neutralised pneumolysin ${ }^{38}$. The results from these supernatant swap experiments suggest that therapies that target pneumolysin may have significant therapeutic potential in cases of severe pneumococcal pneumonia, where risk of bacterial dissemination into the blood is high. Induction of neutralising anti-toxin antibodies via vaccination with a pneumolysin- or toxoid-containing vaccine may also prevent the key tissue-invasive steps that lead to severe manifestations of disease ${ }^{47,48}$. 


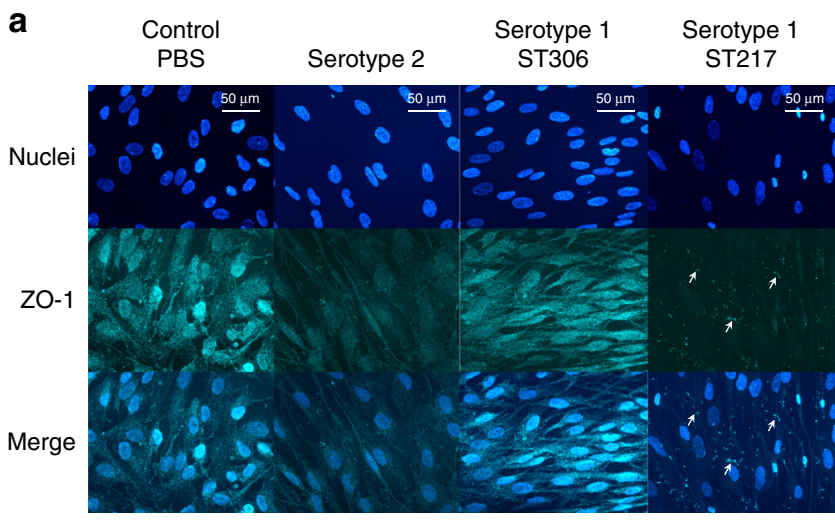

b

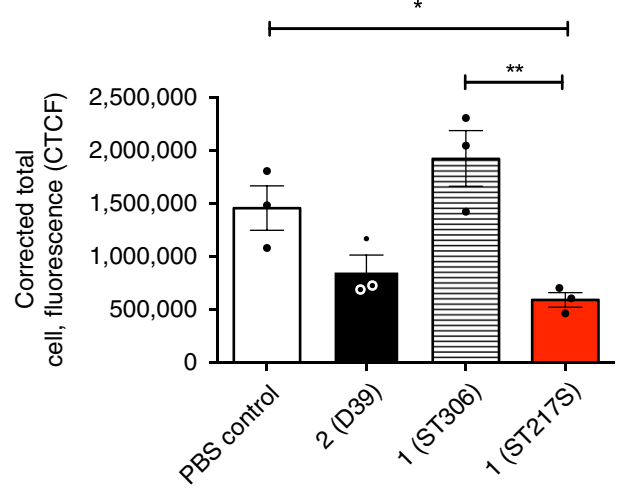

Fig. 9 Pneumococcal infection causes degradation of cellular tight junctions. a Fluorescent image of Human pulmonary alveolar epithelial cells (HPAEpiC) stained with an antibody against zonula occludens (ZO-1). Cells were co-incubated for $12 \mathrm{~h}$ with phosphate buffer saline (PBS), serotype 2 (D39), serotype 1 (sequence type 306 ) and serotype 1 (sequence type 217) at an MOI of bacteria cells $-10: 1$. Scale $=50 \mu \mathrm{m}$. b Corrected Total Cell Fluorescence (CTCF) of HPAEpiCs tagged with an antibody against ZO-1. CTCF was calculated by selecting six cells from each merged image. Integrated density, the total area of the cell, and the mean fluorescence of background readings were used to acquire total fluorescence. Results from triplicate experiments shown and error bars represent mean \pm SEM.

Statisitcal analysis performed using one-way ANOVA and Tukey's multiple comparison test: ${ }^{\star} P$-value $=0.0460$ and ${ }^{\star \star} P$ value $=0.0045$. Source data are provided as a Source Data file.

The results presented here offer insight into why serotype 1 isolates have a high propensity to cause invasive disease. Our findings demonstrate the key relationship between the amount of toxin produced and its release rate as a determinant of serotype 1 invasiveness and pathogenicity.

Our findings also have implications for our overall understanding of pneumococcal disease pathogenesis, whereby high attack rates may in part result from production of large quantities of pneumolysin, quickly released by rapid autolysis. This highlights the significance of pneumolysin for consideration in future vaccine design and as a key target for new adjunctive therapies.

\section{Methods}

Ethics statement. This study was performed in strict accordance with UK Home Office guidelines. Animal experiments were performed at the University of Liverpool and were approved by the local animal welfare and ethics committee.

Mice. For all experiments, 6-10-week-old female BALB/c mice were purchased from Harlan Laboratories (Bicester, UK), and allowed to acclimatise for 7 days prior to use. The animals were housed in the animal facilities under the following conditions; temperature was $21-23^{\circ} \mathrm{C}$ and humidity set at $55-65 \%$. Mice were placed in individually ventilated cages (IVC) from Technoplast (GM500). Automatic watering provided reverse osmosis water sterilised by UV radiation and enrichment included nesting material, balcony, dome home and handling tunnel.

Bacteria. Pneumococci were cultured on blood agar base containing $5 \% \mathrm{vol} / \mathrm{vol}$ horse blood overnight at $37^{\circ} \mathrm{C} 5 \% \mathrm{CO}_{2}$. Single colonies of pneumococci were isolated and cultured in brain heart infusion broth (Oxoid, UK) containing 20\% (vol/vol) fetal bovine serum until required optical density was reached ${ }^{27}$. See Supplementary Table 2 for details of all S. pneumoniae serotypes used in this study.

Construction of pneumococcal D39:ST217ply mutant. Generation of competen S. pneumoniae D39 cells and subsequent transformation were performed using complete transformation medium $(\mathrm{C}+\mathrm{Y}$ media, $\mathrm{pH}=6.8) \operatorname{method}^{49}$. D39 expressing serotype 1 pneumolysin was constructed by replacing the wild-type D39-ply open reading frame with a cassette composed of the serotype 1-ply gene followed by the aphA3 gene (conferring kanamycin resistance) and subsequent selection of kanamycin-resistant transformants (D39:ST217ply-aphA3) on blood agar base medium supplemented with kanamycin. Transformants were verified by Sanger sequencing. The primers used for mutant construction were: Ply-up-for gatgagcgcgacccagtgccag, Ply-up-rev cagatatccgcagagagatcatcgc, Ply-down-for tggatcctgcttgagtttatctcttgcctagcg, Ply-down-rev gggcttgtttagcacggtcgataac, aphA3for ctctgcggatatctgtcgctagtattaaatgc, aphA3-rev tcaagcaggatccatcgatac.

Preparation of challenge dose. Aliquots of $S$. pneumoniae were stored at $-70^{\circ} \mathrm{C}$. When required, suspension was thawed at room temperature and bacteria were harvested by centrifugation before being resuspended in sterile phosphate-buffered saline (PBS)

Infection of mice. Animals were anesthetised with a mixture of $\mathrm{O}_{2}$ and Isofluorane and infected intranasally with $1 \times 10^{6} \mathrm{CFU}$ S. pneumoniae in $50 \mu \mathrm{l}$ of PBS as described previously $\mathrm{y}^{27}$. For nasopharyngeal carriage models, inoculation dose was reduced to $1 \times 10^{5} \mathrm{CFU}$ in $10 \mu \mathrm{l}$ of $\mathrm{PBS}^{50}$. Mice were periodically scored for clinical signs of disease and culled when they became moderately lethargic or else at predetermined times post infection. Signs of disease were based on the scheme of Morton $^{51}$. Mice were culled by cervical dislocation and lungs were removed and prepared for assessment of bacterial colony-forming units. Blood samples were taken from tail bleeds or cardiac puncture under terminal anaesthesia.

Determination of bacterial numbers in nasopharynx, lung and blood. Viable counts of bacteria in lung, nasopharynx, olfactory tissues, brain and blood samples were determined by serial dilution in sterile 1x PBS and plating on blood agar containing $5 \%(\mathrm{v} / \mathrm{v})$ defibinated horse blood and $40 \mu \mathrm{g}$ per $\mathrm{ml}$ of Gentamycin (Sigma). Plates were incubated overnight at $37^{\circ} \mathrm{C} 5 \%(\mathrm{v} / \mathrm{v}) \mathrm{CO}_{2}$ and bacterial colony numbers were assessed the following day.

Tissue preparation (murine lungs). Lung tissue was harvested, weighed, placed into a petri dish and then cut into smaller pieces using a scalpel blade. To help release immune cells via enzymatic digestion, lung tissue was placed in $1.5 \mathrm{ml}$ Eppendorf tubes containing $1 \mathrm{ml}$ of PBS and $10 \mathrm{mg}$ per $\mathrm{ml}$ of Collagenase D (Roche). The Eppendorf tubes were then incubated at $37^{\circ} \mathrm{C}$ for $30 \mathrm{~min}$. After digestion, tissue was passed through a $40-\mu \mathrm{m}$ cell strainer (BD Biosciences) and washed with sterile PBS to create a single-cell suspension. Cell suspensions were then centrifuged at $400 \times g$ for $5 \mathrm{~min}$. The cell pellet was resuspended in 1x Red blood cell lysis buffer (Sigma) to lyse all red blood cells. The cell suspensions were then centrifuged at $400 \times g$ for $5 \mathrm{~min}$ and cell pellet resuspended in cryopreservation media, for storage at $-80^{\circ} \mathrm{C}$. When needed, aliquots of cells were thawed quickly in the water bath.

Tissue preparation (murine nasopharynx). Nasopharyngeal tissue was harvested and placed into bijou tubes containing $3 \mathrm{ml}$ of sterile PBS. The tissue was then mechanically disrupted for $\sim 1$ min using a homogenizer (IKA T10). The homogenised tissue was then passed through a $40-\mu \mathrm{m}$ pore cell strainer and centrifuged at $400 \times g$ for $5 \mathrm{~min}$. The cell pellet was either resuspended in cryopreservation media or used for flow cytometry analysis on the same day.

Flow cytometry. Nasopharynx and lung tissue were collected and prepared as described above. For staining and acquisition, samples were either thawed or used fresh from dissection. Cells were incubated with a 1 in 200 dilution of purified antiCD16/CD32 Fc blocking antibody (eBiosciences) for $30 \mathrm{~min}$ at room temperature. Following incubation with blocking antibody, cell surface markers were stained for An intracellular monoclonal antibody panel was used to detect both intracellular cytokines and transcription factors for different $\mathrm{CD} 4^{+} \mathrm{T}$ cell subsets. The samples were acquired using a BD FACSCanto flow cytometer (BD Biosciences). See supplementary Table 3 for details of antibody panels used. Supplementary Figs. 9-11 show the gating strategies used to idenitify immune cells of interest. 
Serotype 1 infected lungs

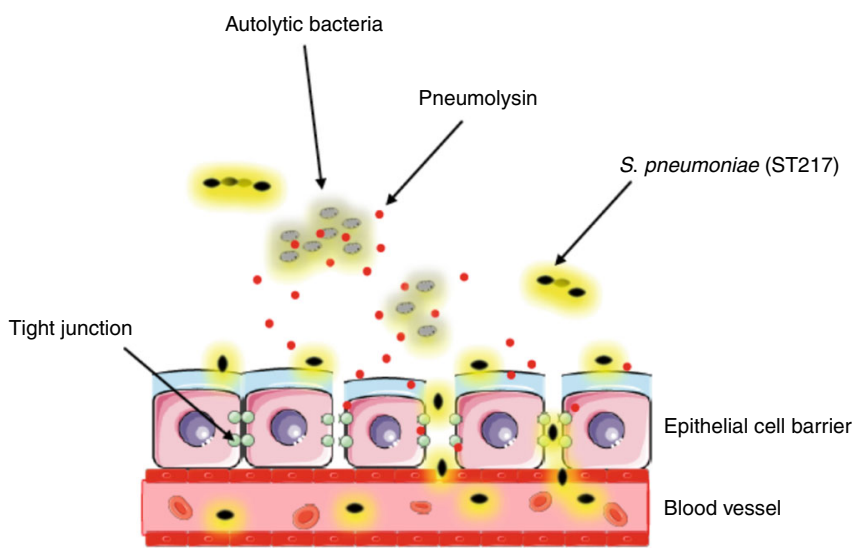

S. pneumoniae infected lungs

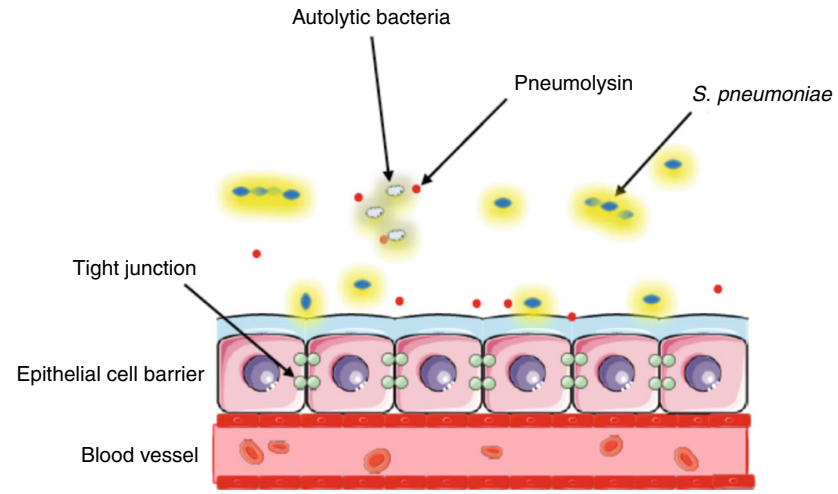

Fig. 10 Comparison of pneumococcal pneumonia in serotype 1 compared to serotype $\mathbf{2}$ infection. In a serotype 1 (ST217/ST3081) infected lung, levels of bacterial lysis are high, resulting in release of high concentrations of the toxin pneumolysin. These high levels of pneumolysin cause damage to tight junctions between epithelial cells and levels of cell cytotoxicity are high. Subsequent damage to lung epithelial cell barriers allows for serotype 1 pneumococci to disseminate from the lungs into the bloodstream and cause bacteraemia. In pneumonia caused by other S. pneumoniae serotypes such as 2 (D39), rates of bacterial lysis are lower and therefore lower concentrations of pneumolysin are released into the lung. This results in lower cell cytotoxicity and reduced damage to cell barriers between lungs and blood, allowing for containment of bacteria within the lungs and therefore no development of bacteraemia.

Autolysis assay. Triton X-100 induced autolysis assays were performed in triplicate as previously described, with the following modifications ${ }^{52}$. Cultures of $S$. pneumoniae were subcultured into $20 \mathrm{ml}$ of fresh $\mathrm{BHI}$ broth and incubated at $37^{\circ} \mathrm{C}$ and $200 \mathrm{rpm}$ until an approximate $A_{600}$ of one was obtained. Cultures were centrifuged for $10 \mathrm{~min}$ at maximum speed. Pellets were resuspended in $1 \mathrm{ml}$ of fresh PBS and the $A_{600}$ was adjusted to 1 in $1 \mathrm{ml}$ of PBS containing $0.01 \%$ Triton X-100 (Sigma) in a cuvette. Cuvettes were covered in parafilm and samples vortexed for $10 \mathrm{~s}$ and the $A_{600}$ of the culture at time zero was recorded. Cultures were then incubated at $37^{\circ} \mathrm{C}$ and $200 \mathrm{rpm}$ for $3 \mathrm{~h}$ and the $A_{600}$ was measured every $15 \mathrm{~min}$. Triton X-100 induced autolysis was presented as a percentage of the initial $A_{600}$ at time zero.

In vitro lung epithelial cell infection. Human lung adenocarcinoma epithelial cell line, A549 (Sigma) and human primary pulmonary alveolar epithelial cells (acquired from ScienCell Research Laboratories - Cat. No 3200) were used to assess cellular damage, disruption to epithelial cell tight junctions and cytokine responses to infection with $S$. pneumoniae. All cells were tested for mycoplasma contamination prior to starting experiments. A549 cells were maintained in DMEM supplemented with $10 \%$ foetal bovine serum whilst Alveolar Epithelial Cell Medium (AECM) was used for human primary pulmonary alveolar epithelial cells. In all, $500 \mu \mathrm{l}$ of cells at $2 \times 10^{5}$ cells per ml density were seeded onto Greiner Thincerts (surface area $0.94 \mathrm{~cm}^{2}, 3 \mu \mathrm{m}$ pore diameter). Inserts were placed in 12well tissue culture plates (Greiner) and incubated in $1.5 \mathrm{ml}$ of appropriate cell culture media at $37^{\circ} \mathrm{C}, 5 \% \mathrm{CO}_{2}$ for 2 days. Frozen bacterial stocks were thawed, and suspended to a desired final concentration in DMEM $+10 \%$ FBS or AECM before addition to tissue culture inserts.

Trans Epithelial Electrical Resistance measurement. Trans Epithelial Electrical Resistance measurement (TEER) was measured in Ohms using an EVOM2 Epithelial Voltohmmeter (WPI). Wells without cells were used as blank standard. Measurements were done in triplicate and the mean was calculated and blank subtracted. Blank corrected resistance values were multiplied by 0.94 (the surface area of insert) give TEER in $\mathrm{Ohms} \times \mathrm{cm}^{2}$.

Immunocytochemistry. Briefly, coverslips containing human primary pulmonary alveolar epithelial cells (HPAEpiCs) were washed twice with phosphate-buffered saline (PBS, pH 7.6) and fixed with fresh high-grade $4 \%$ paraformaldehyde (PFA) for $10 \mathrm{~min}$. The PFA was aspirated and the coverslips were washed 4 times in PBS for 5 min each time. The coverslips were blocked with 1.5\% Normal Goat Serum (NGS, Sigma Aldrich UK) for $2 \mathrm{~h}$ at room temperature. Primary antibody incubation was carried out for $2 \mathrm{~h}$ at room temperature or overnight at $4{ }^{\circ} \mathrm{C}$ with gentle agitation. HPAEpiCs were identified by using a Zonula Occludens primary antibody (Rb polyclonal anti ZO-1 - Abcam ab96587). ZO-1 antibody was used in a 1:100 dilution and the samples were washed in PBS for $5 \times 5 \mathrm{~min}$. The corresponding secondary antibody (goat anti-rabbit IgG Abcam ab96883) conjugated to Dylight $^{\circ}(1: 1000)$ was added to the cells for $2 \mathrm{~h}$ at room temperature with gentle agitation. Cells were washed in PBS for $5 \times 5 \mathrm{~min}$, and the coverslips were inverted onto a drop of mounting medium containing DAPI (Fluoroshield ${ }^{\mathrm{TM}}$ Sigma Aldrich UK) on a microscope slide, and stored at $4{ }^{\circ} \mathrm{C}$. The immunostained cells were viewed under fluorescence (Nikon Eclipse 80i fluorescent microscope) with the appropriate excitations for each fluorophore. All images were analysed using ImageJ-Win64.

Measuring cell fluorescence. To determine the level of cellular fluorescence using microscopy, ImageJ-Win64 software was used as described previously ${ }^{53}$. In brief, an outline was drawn around each cell to measure the area and mean fluorescence, along with several adjacent background readings. The corrected total cell fluorescence was calculated as follows:

$$
\begin{aligned}
(\mathrm{CTCF})= & \text { integrated density } \\
& -(\text { area of selected cells } \times \text { mean fluorescence of background readings })
\end{aligned}
$$

Alveolar permeability assays. Transwell ThinCerts ${ }^{\mathrm{TM}}$ (pore size $3.0 \mu \mathrm{m}, 8.4 \mathrm{~mm}$ diameter, polyester membrane, Greiner Bio-One, Stonehouse, UK) were coated with Poly-D-Lysine Hydrobromide (Sigma Aldrich, UK). The lower compartments of the Transwell chambers were filled with $1.5 \mathrm{ml}$ Alveolar Epithilial Cell Medium (AECM). HPAEpiCs in $500 \mu \mathrm{l}$ AECM $\left(5 \times 10^{4}\right.$ cells per well $)$ were seeded on the Thincert's membrane and reached confluency $24 \mathrm{~h}$ post seeding. Cells were coincubated with the appropriate pneumococcal serotype for 6,12 and $24 \mathrm{~h}$ before fluorescein isothiocyanate (FITC)-dextran $(70 \mathrm{kDa}, 1 \mathrm{mg} / \mathrm{ml}$; Sigma-Aldrich, Irvine, UK) was added to the apical side. In all, $100 \mu \mathrm{l}$ samples were collected at 0 and $60 \mathrm{~min}$ after the FITC addition from the top and the lower compartment. Our Thincert setup was gently agitated for the duration of the permeability assay. Fluorescence (ex: $485 \mathrm{~nm}$; em: $535 \mathrm{~nm}$ ) was measured using a BMG Omega fluorescent plate reader. The mean fluorescence recorded from the lower compartment of naive cells at the collection point of FITC $(60 \mathrm{~min})$ was given the value of 1 . Changes to the experimental Thincerts is compared against the control FITC levels. The absolute permeability P [ $\mathrm{cm}$ per s] was calculated by the following equation ${ }^{54}$ :

$$
\mathrm{P}=\left[\mathrm{C}_{\mathrm{t}}-\mathrm{C}_{\mathrm{t} 0}\right] \times \mathrm{V} / \mathrm{A} \times \mathrm{t} \times \mathrm{C}_{0}
$$

$C(\mathrm{t})$ is the FITC solution found on the lower compartment ( $\mu \mathrm{g}$ per $\mathrm{ml}$ ), $60 \mathrm{~min}$ after the solution was added to the Thincerts, $C\left(\mathrm{t}_{0}\right)$ is the concentration of FITC solution at $0 \mathrm{~min}, t$ is the duration of the flux (seconds), $V$ is the volume of the solution found in the lower compartment $\left(\mathrm{cm}^{3}\right), A$ is the surface of the Thincert membrane $\left(33 \mathrm{~mm}^{2}\right)$ and $C_{0}$ is the original concentration of the FITC solution added on the apical side ( $\mu \mathrm{g}$ per $\mathrm{ml}$ ). FITC concentrations were calculated in accordance to a FITC-dextran standard curve.

Cell viability assays. Prior to the assay, 96-well flat-bottomed micro-plates were coated with $15 \%$ Poly-D Lysine Hydrobromide solution, and seeded with $5 \times 10^{4}$ cells per well (total cell media volume of $200 \mu \mathrm{l}$ per well). The micro-plates were incubated at $37^{\circ} \mathrm{C} / 5 \% \mathrm{CO}_{2}$ for $24 \mathrm{~h}$. The supernatant was carefully removed and the cells were washed with $100 \mu \mathrm{l}$ 1xPBS.

3-(4,5-Dimethylthiazol-2-yl)-2,5-diphenyltetrazolium bromide assay. The MTT assay measures the reduction of MTT (3-(4,5-Dimethylthiazol-2-yl)-2,5diphenyltetrazolium bromide) to formazan within living cells. MTT solution was 
prepared by the addition of $1 \mathrm{mg}$ MTT (Sigma Aldrich ${ }^{\oplus}$ ) to $1 \mathrm{ml}$ of phenol red-free media $\left(\mathrm{Gibco}^{\circledast}\right)$.

Cell culture media was removed and wells were washed with $100 \mu$ PBS followed by the addition of $100 \mu \mathrm{l}$ MTT $(1 \mathrm{mg}$ per ml) per well protected from light. The plate supplemented with MTT was incubated at $37^{\circ} \mathrm{C} / 5 \% \mathrm{CO}_{2}$ for $4 \mathrm{~h}$. Following incubation, light microscopy was used to observe whether formazan crystals had formed within the cells. Then the supernatant was removed by aspiration and $100 \mu \mathrm{l}$ of isopropanol was added to the wells to dissolve the crystals formed. Colour change was assessed by absorbance at $560 \mathrm{~nm}$ using a ThermoScientific Multiskan plate-reader with the SkanIt (Research Edition) for Multiskan Spectrum 2.2 software.

Liposomes. Cholesterol and sphingomyelin liposomes were obtained from egg yolk (Sigma Aldrich). Lipids were individually dissolved in chloroform at $1 \mathrm{mg}$ per $\mathrm{ml}$ concentrations. For preparation of liposomes, individual lipids were mixed to form Cholesterol:sphingomyelin ( $66 \mathrm{~mol} / \% \mathrm{cholesterol})$ and sphingomyelin-only liposomes. Chloroform was completely evaporated with nitrogen gas for $30 \mathrm{~min}$, followed by hydration with PBS (ThermoFisher Scientific). Following incubation for $30 \mathrm{~min}$ at $45^{\circ} \mathrm{C}$ in an Eppendorf thermomixer with vortexing, liposome preparations were sonicated for $30 \mathrm{~min}$ at $4{ }^{\circ} \mathrm{C}$. Final concentration of liposomes was $2 \mathrm{mg} / \mathrm{ml}$.

Pneumolysin ELISA. ELISAs were used to detect the amount of pneumolysin produced by different serotypes of $S$. pneumoniae $\left(10^{7} \mathrm{CFU}\right.$ per $\left.\mathrm{ml}\right)$ when bacteria were lysed with penicillin/streptomycin antibiotics over the course of an hour at room temperature. Bacterial CFU counts were performed to check full lysis with antibiotics had occurred. In addition, all isolates were checked for penicillin resistance (Supplementary Fig. 8) The ELISA was also used to detect the amount of pneumolysin released into the supernatant when $10^{7} \mathrm{CFU}$ per $\mathrm{ml}$ of $S$. pneumoniae when cultured in PBS for 45 min. ELISA plates were coated with $1 \mu \mathrm{g}$ per well of IgG1-Ply4 (Abcam 71810) and incubated overnight at $4{ }^{\circ} \mathrm{C}$ before washing and blocking with Peprotech Blocking Buffer (1\% BSA in PBS). Purified recombinant Ply (allele 1, D39) of various concentrations was included as the standard curve in the assay (2-fold dilutions from $400 \mathrm{ng} / \mathrm{ml}$ to $3.125 \mathrm{ng} / \mathrm{ml}$ ), which was incubated for $1 \mathrm{~h}$ at $37^{\circ} \mathrm{C}$. After samples were added and incubated, $1 \mu \mathrm{g}$ per $\mathrm{ml}$ of detection antibody (Rabbit polyclonal to PLY antibody, Abcam ab71811) was added to the wells and incubated for $2 \mathrm{~h}$ at room temperature. Goat anti-rabbit IgG alkaline phosphatase (Abcam Ab97048) (1:5000 dilution) was added to wells for $30 \mathrm{~min}$ at room temperature. After washing, the colour reagent Alkaline Phosphatase yellow pNNP (Sigma p7998) was added and plate incubated for $30 \mathrm{~min}$ in the dark. The amount of pneumolysin present was assessed by measuring the plate at $405 \mathrm{~nm}$ with an ELISA plate reader. Recorded values for the recombinant pneumolysin were used to plot a curve of OD405 vs ng/ml pneumolysin concentration and test samples were quantified in $\mathrm{ng}$ per $\mathrm{ml}$ using the equation of the curve.

Haemolytic assay. In all, $4 \%$ red blood cell solution was prepared by adding $400 \mu \mathrm{l}$ of sheep blood pellet to $10 \mathrm{ml}$ of PBS. Bacterial stocks were thawed, centrifuged and resuspended to $1 \times 10^{7} \mathrm{CFU}$ per $\mathrm{ml}$. Bacteria were lysed using Penicillin and Streptomycin antibiotics at room temperature for $30 \mathrm{~min}$ and Miles and Misra counts were performed to check for $100 \%$ lysis (Supplementary Fig. 8). A 1:1 ratio of lysed bacteria to $4 \%$ RBC solution was added to a microplate before bacteria was diluted twofold with $4 \% \mathrm{RBC}$ solution. After 30 min incubation the plate was centrifuged and supernatant was removed from each well and placed in a microplate. The OD was measured at $540 \mathrm{~nm}$ on a spectrometer to determine the levels of haemoglobin released.

Statistics. Statistical analysis was carried out using the GraphPad Prism $6^{\oplus}$ version 5 statistical package (GraphPad Software, Inc). The statistical significance according to the $P$-values were summarised as follows: ${ }^{*} P$-value $<0.05$, ${ }^{* *} P$-value $<0.01$, ${ }^{* * *} P$-value $<0.001$ and $* * * * P$-value $<0.0001$. Reported measurements are from distinct samples and not repeated measures.

Reporting summary. Further information on research design is available in the Nature Research Reporting Summary linked to this article.

\section{Data availability}

The source data underlying Figs. 1, 3-9, and Supplementary Figs. 1-5, are provided as a Source Data file.

Received: 22 January 2019; Accepted: 23 March 2020;

Published online: 20 April 2020

\section{References}

1. O'Brien, K. L. et al. Burden of disease caused by Streptococcus pneumoniae in children younger than 5 years: global estimates. Lancet 374, 893-902 (2009).
2. Ortqvist, A., Hedlund, J. \& Kalin, M. Streptococcus pneumoniae epidemiology, risk factors, and clinical features. Semin. Respir. Crit. Care Med. 26, 563-574 (2005)

3. Ritchie, N. D., Mitchell, T. J. \& Evans, T. J. What is different about serotype 1 pneumococci? Future Microbiol. 7, 33-46 (2012).

4. Chaguza, C. et al. Population genetic structure, antibiotic resistance, capsule switching and evolution of invasive pneumococci before conjugate vaccination in Malawi. Vaccine 35, 4594-4602 (2017).

5. Brueggemann, A. B. \& Spratt, B. G. Geographic distribution and clonal diversity of Streptococcus pneumoniae serotype 1 isolates. J. Clin. Microbiol. 41, 4966-4970 (2003).

6. Chaguza, C. et al. Understanding pneumococcal serotype 1 biology through population genomic analysis. BMC Infect. Dis. 16, 649 (2016).

7. Brueggemann, A. B. et al. Temporal and geographic stability of the serogroupspecific invasive disease potential of Streptococcus pneumoniae in children. $J$. Infect. Dis. 190, 1203-1211 (2004)

8. Jones, N., Huebner, R., Khoosal, M., Crewe-Brown, H. \& Klugman, K. The impact of HIV on Streptococcus pneumoniae bacteraemia in a South African population. AIDS 12, 2177-2184 (1998).

9. Scott, J. A. et al. Serotype distribution and prevalence of resistance to benzylpenicillin in three representative populations of Streptococcus pneumoniae isolates from the coast of Kenya. Clin. Infect. Dis. 27, 1442-1450 (1998).

10. Hausdorff, W. P. The roles of pneumococcal serotypes 1 and 5 in paediatric invasive disease. Vaccine 25, 2406-2412 (2007).

11. du Plessis, M. et al. Phylogenetic analysis of invasive serotype 1 pneumococcus in South Africa, 1989 to 2013. J. Clin. Microbiol. 54, 1326-1334 (2016).

12. Canvin, J. R. et al. The role of pneumolysin and autolysin in the pathology of pneumonia and septicemia in mice infected with a type 2 pneumococcus. $J$. Infect. Dis. 172, 119-123 (1995)

13. Boulnois, G. J., Paton, J. C., Mitchell, T. J. \& Andrew, P. W. Structure and function of pneumolysin, the multifunctional, thiol-activated toxin of Streptococcus pneumoniae. Mol. Microbiol. 5, 2611-2616 (1991).

14. Garcia-Suarez Mdel, M. et al. The role of pneumolysin in mediating lung damage in a lethal pneumococcal pneumonia murine model. Respir. Res. 8, 3, (2007).

15. Zysk, G. et al. Pneumolysin is the main inducer of cytotoxicity to brain microvascular endothelial cells caused by Streptococcus pneumoniae. Infect. Immun. 69, 845-852 (2001).

16. Rubins, J. B. et al. Pneumolysin in pneumococcal adherence and colonization Microb. Pathog. 25, 337-342 (1998).

17. Tweten, R. K. Cholesterol-dependent cytolysins, a family of versatile poreforming toxins. Infect. Immun. 73, 6199-6209 (2005).

18. McNeela, E. A. et al. Pneumolysin activates the NLRP3 inflammasome and promotes proinflammatory cytokines independently of TLR4. PLoS Pathog. 6 , e1001191, (2010)

19. Rai, P., He, F., Kwang, J., Engelward, B. P. \& Chow, V. T. Pneumococcal pneumolysin induces DNA damage and cell cycle arrest. Sci. Rep. 6, 22972, (2016).

20. Fatykhova, D. et al. Serotype 1 and 8 pneumococci evade sensing by inflammasomes in human lung tissue. PLoS One 10, e0137108 (2015).

21. Walker, J. A., Allen, R. L., Falmagne, P., Johnson, M. K. \& Boulnois, G. J. Molecular cloning, characterization, and complete nucleotide sequence of the gene for pneumolysin, the sulfhydryl-activated toxin of Streptococcus pneumoniae. Infect. Immun. 55, 1184-1189 (1987).

22. Lopez, R., Garcia, E., Garcia, P. \& Garcia, J. L. The pneumococcal cell wall degrading enzymes: a modular design to create new lysins? Microb. Drug Resist. 3, 199-211 (1997).

23. Price, K. E. \& Camilli, A. Pneumolysin localizes to the cell wall of Streptococcus pneumoniae. J. Bacteriol. 191, 2163-2168 (2009).

24. Price, K. E., Greene, N. G. \& Camilli, A. Export requirements of pneumolysin in Streptococcus pneumoniae. J. Bacteriol. 194, 3651-3660 (2012).

25. Hirst, R. A. et al. Streptococcus pneumoniae deficient in pneumolysin or autolysin has reduced virulence in meningitis. J. Infect. Dis. 197, 744-751 (2008).

26. Berry, A. M. et al. Effect of defined point mutations in the pneumolysin gene on the virulence of Streptococcus pneumoniae. Infect. Immun. 63, 1969-1974 (1995).

27. Kadioglu, A. et al. Host cellular immune response to pneumococcal lung infection in mice. Infect. Immun. 68, 492-501 (2000).

28. Berry, A. M., Lock, R. A., Hansman, D. \& Paton, J. C. Contribution of autolysin to virulence of Streptococcus pneumoniae. Infect. Immun. 57, 2324-2330 (1989).

29. Feldman, C. et al. Pneumolysin induces the salient histologic features of pneumococcal infection in the rat lung in vivo. Am. J. Respir. Cell Mol. Biol. 5, 416-423 (1991).

30. Rubins, J. B. et al. Dual function of pneumolysin in the early pathogenesis of murine pneumococcal pneumonia. J. Clin. Invest. 95, 142-150 (1995). 
31. Miller, E., Andrews, N. J., Waight, P. A., Slack, M. P. \& George, R. C. Effectiveness of the new serotypes in the 13-valent pneumococcal conjugate vaccine. Vaccine 29, 9127-9131 (2011).

32. Gingles, N. A. et al. Role of genetic resistance in invasive pneumococcal infection: identification and study of susceptibility and resistance in inbred mouse strains. Infect. Immun. 69, 426-434 (2001).

33. Denny, P. et al. A major locus conferring susceptibility to infection by Streptococcus pneumoniae in mice. Mamm. Genome 14, 448-453 (2003).

34. Neill, D. R. et al. T regulatory cells control susceptibility to invasive pneumococcal pneumonia in mice. PLoS Pathog. 8, e1002660, (2012).

35. van Ginkel, F. W. et al. Pneumococcal carriage results in ganglioside-mediated olfactory tissue infection. Proc. Natl Acad. Sci. USA 100, 14363-14367 (2003).

36. Paradiso, P. R. Advances in pneumococcal disease prevention: 13-valent pneumococcal conjugate vaccine for infants and children. Clin. Infect. Dis. 52, 1241-1247 (2011).

37. Martner, A., Dahlgren, C., Paton, J. C. \& Wold, A. E. Pneumolysin released during Streptococcus pneumoniae autolysis is a potent activator of intracellular oxygen radical production in neutrophils. Infect. Immun. 76, 4079-4087 (2008).

38. Henry, B. D. et al. Engineered liposomes sequester bacterial exotoxins and protect from severe invasive infections in mice. Nat. Biotechnol. 33, 81-88 (2015).

39. Lehmann, A. D. et al. An in vitro triple cell co-culture model with primary cells mimicking the human alveolar epithelial barrier. Eur. J. Pharm. Biopharm. 77, 398-406 (2011).

40. Anderson, J. M., Stevenson, B. R., Jesaitis, L. A., Goodenough, D. A. \& Mooseker, M. S. Characterization of ZO-1, a protein component of the tight junction from mouse liver and Madin-Darby canine kidney cells. J. Cell Biol. 106, 1141-1149 (1988).

41. Jonczyk, M. S. et al. Variation in inflammatory response during Pneumococcal infection is influenced by host-pathogen interactions but associated with animal survival. Infect. Immun. 84, 894-905 (2016).

42. Wood, W. B. Studies on the mechanism of recovery in Pneumococcal Pneumonia: I. The action of type specific antibody upon the pulmonary lesion of experimental pneumonia. J. Exp. Med. 73, 201-222 (1941).

43. Rubins, J. B., Duane, P. G., Charboneau, D. \& Janoff, E. N. Toxicity of pneumolysin to pulmonary endothelial cells in vitro. Infect. Immun. 60 , 1740-1746 (1992).

44. Stevenson, B. R., Siliciano, J. D., Mooseker, M. S. \& Goodenough, D. A. Identification of ZO-1: a high molecular weight polypeptide associated with the tight junction (zonula occludens) in a variety of epithelia. J. Cell Biol. 103, 755-766 (1986).

45. Jusot, J. F. et al. Airborne dust and high temperatures are risk factors for invasive bacterial disease. J. Allergy Clin. Immunol. https://doi.org/10.1016/j. jaci.2016.04.062 (2016).

46. Gessner, B. D., Mueller, J. E. \& Yaro, S. African meningitis belt pneumococcal disease epidemiology indicates a need for an effective serotype 1 containing vaccine, including for older children and adults. BMC Infect. Dis. 10, 22, (2010).

47. Mann, B. et al. Broadly protective protein-based pneumococcal vaccine composed of pneumolysin toxoid-CbpA peptide recombinant fusion protein. J. Infect. Dis. 209, 1116-1125 (2014).

48. Hermand, P. et al. Preclinical evaluation of a chemically detoxified pneumolysin as pneumococcal vaccine antigen. Hum. Vaccin. Immunother. 13, 220-228 (2017).

49. Lacks, S. \& Hotchkiss, R. D. Formation of amylomaltase after genetic transformation of pneumococcus. Biochim. Biophys. Acta 45, 155-163 (1960).

50. Richards, L., Ferreira, D. M., Miyaji, E. N., Andrew, P. W. \& Kadioglu, A. The immunising effect of pneumococcal nasopharyngeal colonisation; protection against future colonisation and fatal invasive disease. Immunobiology 215, 251-263 (2010).

51. Morton, D. B. \& Griffiths, P. H. Guidelines on the recognition of pain, distress and discomfort in experimental animals and an hypothesis for assessment. Vet. Rec. 116, 431-436 (1985).
52. Bose, J. L., Lehman, M. K., Fey, P. D. \& Bayles, K. W. Contribution of the Staphylococcus aureus Atl AM and GL murein hydrolase activities in cell division, autolysis, and biofilm formation. PLoS One 7, e42244, (2012).

53. McCloy, R. A. et al. Partial inhibition of Cdk1 in $\mathrm{G} 2$ phase overrides the SAC and decouples mitotic events. Cell Cycle 13, 1400-1412 (2014).

54. Bischoff, I. et al. Pitfalls in assessing microvascular endothelial barrier function: impedance-based devices versus the classic macromolecular tracer assay. Sci. Rep. 6, 23671 (2016).

\section{Acknowledgements}

The study was supported by funding from a UK Medical Research Council Programme Grant (MR/P011284/1) awarded to A.K.; L.C.J. was supported by a University of Liverpool, Institute of Infection and Global Health funded PhD studentship, part funded by the University of Warwick, WCPRS programme. D.R.N. is supported by a Sir Henry Dale Fellowship jointly funded by the Wellcome Trust and the Royal Society (Grant number 204457/Z/16/Z).

\section{Author contributions}

L.C.J., S.P., M.B., D.R.N. and A.K. planned and designed the experiments; L.C.J., S.P., S.K., M.B., R.X. and L.B.M. performed the experiments; L.C.J., S.P., M.S., M.Y., M.B., D.R.N., C.G.D., D.B.E. and A.K. analysed the data; C.G.D., D.B.E., D.R.N. and A.K. provided reagents and materials. L.C.J., D.R.N. and A.K. wrote the manuscript. C.G.D., D.B.E. and A.K. supervised the study. All authors read and approved the final version of the manuscript.

\section{Competing interests}

The authors declare no competing interests.

\section{Additional information}

Supplementary information is available for this paper at https://doi.org/10.1038/s41467020-15751-6.

Correspondence and requests for materials should be addressed to A.K.

Peer review information Nature Communications thanks Sven Hammerschmidt and the other, anonymous, reviewer(s) for their contribution to the peer review of this work.

Reprints and permission information is available at http://www.nature.com/reprints

Publisher's note Springer Nature remains neutral with regard to jurisdictional claims in published maps and institutional affiliations.

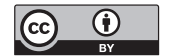

Open Access This article is licensed under a Creative Commons Attribution 4.0 International License, which permits use, sharing, adaptation, distribution and reproduction in any medium or format, as long as you give appropriate credit to the original author(s) and the source, provide a link to the Creative Commons license, and indicate if changes were made. The images or other third party material in this article are included in the article's Creative Commons license, unless indicated otherwise in a credit line to the material. If material is not included in the article's Creative Commons license and your intended use is not permitted by statutory regulation or exceeds the permitted use, you will need to obtain permission directly from the copyright holder. To view a copy of this license, visit http://creativecommons.org/ licenses/by/4.0/.

(C) The Author(s) 2020 